\title{
Mer regulates microglial M1/M2 polarization and alleviates neuroinflammation following traumatic brain injury
}

\section{haijian wu}

Zhejiang University School of Medicine Second Affiliated Hospital jingwei zheng

Zhejiang University School of Medicine Second Affiliated Hospital shenbin $\mathrm{xu}$

Zhejiang University School of Medicine Second Affiliated Hospital

\section{yuanjian fang}

Zhejiang University School of Medicine Second Affiliated Hospital

\section{anwen shao}

Zhejiang University School of Medicine Second Affiliated Hospital ligen shi

Zhejiang University School of Medicine Second Affiliated Hospital jianan lu

Zhejiang University School of Medicine Second Affiliated Hospital xiaoyu wang

Zhejiang University School of Medicine Second Affiliated Hospital yirong wang

Zhejiang University School of Medicine Sir Run Run Shaw Hospital zhen zhao

University of Southern California Keck School of Medicine Jianmin Zhang ( $\nabla$ zjm135@zju.edu.cn )

Department of Neurosurgery, The Second Affiliated Hospital, School of Medicine, Zhejiang University https://orcid.org/0000-0002-3184-1502

\section{Research}

Keywords: Mer, Microglia, M1/M2 polarization, neuroinflammation, TBI

Posted Date: May 22nd, 2020

DOI: https://doi.org/10.21203/rs.3.rs-29587/v1 
License: (c) (i) This work is licensed under a Creative Commons Attribution 4.0 International License. Read Full License 
Mer regulates microglial M1/M2 polarization and alleviates neuroinflammation following traumatic brain injury

Haijian $\mathrm{Wu}^{\mathrm{a}, \mathrm{b}, \mathrm{e}, \# \text {, Jingwei Zheng }}{ }^{\mathrm{a}, \#}$, Shenbin Xu ${ }^{\mathrm{a}}$, Yuanjian Fang ${ }^{\mathrm{a}}$, Anwen Shao ${ }^{\mathrm{a}}$, Ligen Shi ${ }^{\mathrm{a}}$, Jianan Lu ${ }^{\mathrm{a}}$, Xiaoyu Wang $^{\mathrm{a}}$, Yirong Wang ${ }^{\mathrm{b}}$, Zhen Zhao ${ }^{\mathrm{e}}$, Jianmin Zhang ${ }^{\mathrm{a}, \mathrm{c}, \mathrm{d}, \uparrow}$

${ }^{\text {a }}$ Department of Neurosurgery, Second Affiliated Hospital, School of Medicine, Zhejiang University, Hangzhou, China

${ }^{\mathrm{b}}$ Department of Neurosurgery, Sir Run Run Shaw Hospital, School of Medicine, Zhejiang University, Hangzhou, Zhejiang, China

${ }^{\mathrm{c}}$ Brain Research Institute, Zhejiang University, Hangzhou, China

${ }^{\mathrm{d}}$ Collaborative Innovation Center for Brain Science, Zhejiang University, Hangzhou, China

${ }^{\mathrm{e}}$ Center for Neurodegeneration and Regeneration, Zilkha Neurogenetic Institute and Department of Physiology and Biophysics, Keck School of Medicine, University of Southern California, Los Angeles, California, USA

${ }^{\#}$ These authors contributed equally to this work.

†Correspondence:

Jianmin Zhang, MD \& Ph.D., Professor and Chair

Department of Neurosurgery, Second Affiliated Hospital, School of Medicine, Zhejiang University, 88 Jiefang Road, Hangzhou 310009 , Zhejiang, China;

E-mail: zjm135@zju.edu.cn; 


\begin{abstract}
Background: Traumatic brain injury (TBI) is a leading cause of death and disability worldwide. Microglial activation and neuroinflammation are key cellular events following TBI, but the regulatory and functional mechanisms are still not well understood. Myeloid-epithelial-reproductive tyrosine kinase (Mer), a member of the Tyro-Axl-Mer (TAM) family of receptor tyrosine kinases, regulates multiple features of microglial physiology. However, its function in regulating the innate immune response and microglial M1/M2 polarization in TBI has not been addressed. The present study aimed to evaluate the role of Mer in regulating microglial M1/M2 polarization and neuroinflammation following TBI.
\end{abstract}

Methods: The controlled cortical impact (CCI) mouse model was established. Mer siRNA was intracerebroventricularly administered and recombinant protein S (PS) was intravenously applied for intervention. The neurobehavioral assessments, RT-PCR, Western blot, immunohistochemistry and confocal microscopy analysis, Nissl and Fluoro-Jade B staining, brain water content measurement, and contusion volume assessment were performed.

Results: Mer is upregulated and regulates microglial M1/M2 polarization and neuroinflammation in the acute stage of TBI. Mechanistically, Mer activates the signal transducer and activator of transcription 1 (STAT1)/suppressor of cytokine signaling 1/3 (SOCS1/3) pathway. Inhibition of Mer markedly decreases microglial M2 polarization while increases M1 polarization, which exacerbates the secondary brain damage and sensorimotor deficits after TBI. Recombinant PS exerts beneficial effects in TBI mice through Mer activation.

Conclusions: Mer is an important regulator of microglial M1/M2 polarization and neuroinflammation, and may be considered as a potential target for therapeutic intervention in TBI.

\title{
Keywords
}

Mer, Microglia, M1/M2 polarization, neuroinflammation, TBI 


\section{Background}

Traumatic brain injury (TBI) is a devastating neurological disease and a major cause of death and disability worldwide, especially among young adults [1]. It is estimated that approximately 70 million individuals suffer a TBI annually [2]. Notably, the pathophysiological processes of TBI are rather complex, which occur in the acute phase and yet continue to evolve over time, leading to persistent, sometimes life-long consequences [3]. TBI is an important risk factor for a variety of chronic neurological disorders, such as epilepsy, stroke, psychiatric illness, and neurodegenerative diseases [4]. There is no doubt that TBI not only severely affects the daily life of survivors and their families, but also results in substantial socio-economic impacts [5]. However, a comprehensive understanding of this notorious disease is still lacking, and the need for therapeutics that effectively improve longterm functional outcomes in TBI survivors remains unmet.

Mechanistically, both primary and secondary injury mechanisms are involved in the neuropathology of TBI. Secondary brain injury, which occurs from initial insults to the brain, is an important determinant of TBI outcomes [6]. It consists of ionic homeostasis disturbance, excitotoxicity, oxidative stress, inflammation, and cell death, among others. In particular, neuroinflammation is one of the most common and important cellular events following TBI [7]. It can evolve over minutes to days, months, and even years after the initial injury, and contributes to the development of both acute and chronic neurological consequences post-insult. Microglia, the major resident immune cells in the brain, are presumed to be key players in neuroinflammation after TBI [8]. It has reported that microglia are highly plastic and assume their functional phenotypes dependent on different microenvironmental cues, such as pro-inflammation, anti-inflammation, among others [9]. The pro-inflammatory M1 phenotype expressing signature markers such as CD16 and CD32 tends to release destructive mediators, including tumor necrosis factor- $\alpha$ (TNF- $\alpha$ ), interleukin- $1 \beta$ (IL-1 $\beta$ ) and inducible nitric oxide synthase (iNOS). In contrast, the anti-inflammatory M2 phenotype, characterized by the molecular signatures of CD206 and arginase 1 (Arg-1), produces beneficial mediators such as interleukin-10 (IL-10) and transforming growth factor- $\beta$ (TGF- $\beta$ ) [10]. Notably, the M1 phenotype is more likely associated with uncontrolled neuroinflammation often observed in neurodegenerative diseases, while the M2 phenotype promotes inflammation resolution and tissue repair [11]. Thus, a balanced response between polarized microglial phenotypes is essential for immune homeostasis in the brain.

Currently, the dualistic roles of distinctly polarized microglial populations have been demonstrated in major central nervous system diseases (CNS), including TBI $[12,13]$. Studies from animal models indicated that despite both M1-like and M2-like polarized microglia being activated after TBI, the activity of M2-like response declines over time, whereas the pathological M1-like effect can persist for a long period of time post-insult [12,13], and modulating the M1-M2 polarization balance has been shown to be beneficial for functional outcomes $[14,15]$. However, the molecular mechanisms underlying microglial polarization in TBI are still not fully understood.

Myeloid-epithelial-reproductive tyrosine kinase (Mer), a member of the Tyro-Axl-Mer (TAM) family of receptor tyrosine kinases [16], plays a critical role in the regulation of inflammatory responses in peripheral macrophages [17-20] and brain microglial cells [21-23]. However, its function in regulating the innate immune response and microglial M1/M2 polarization in TBI has not been addressed. Here, we report Mer upregulation in microglia in the acute stage of TBI is a key modulator of microglial M1/M2 polarization and neuroinflammation in a controlled cortical impact (CCI) mouse model, and inhibition of Mer shifts microglial polarization toward the M1 phenotype via signal transducer and activator of transcription 1 (STAT1)/suppressor of cytokine signaling 1/3 (SOCS1/3) pathway, which exacerbates neuroinflammation and secondary brain damage following TBI. 


\section{Materials and methods}

\section{Animals}

Adult male C57BL/6 mice (22-27 g, aged 8-10 weeks) which were purchased from Slac Laboratory Co., Ltd. (Shanghai, China) were used for this study. All mice were housed in filter-top cages and fed a standard diet, with a 12-h light/dark cycle. Free access to food and water as well as controlled temperature and humidity were provided. All animal experiments were performed according to the Institutional Animal Care and Use Committee of Zhejiang University. The procedures were conducted according to the National Institutes of Health's Guide for the Care and the Use of Laboratory Animals and the ARRIVE (Animal Research: Reporting In Vivo Experiments) guidelines. All efforts were made to minimize animal suffering and the number of animals killed.

\section{Randomization and Blinding}

All animals were randomized for group allocation and surgical procedures, and included in the analysis. The operators responsible for the experimental procedures and data analysis were blinded and unaware of group allocation throughout the experiments.

\section{TBI model}

TBI was induced in C57BL/6 mice using a CCI model (Suppl. Fig. 1A), as described previously [24]. Briefly, mice were anesthetized by an intraperitoneal injection of pentobarbital sodium $(50 \mathrm{mg} / \mathrm{kg})$ and placed in the stereotaxic frame. A 4-mm diameter craniotomy was performed using a portable drill over the right parietal cortex between bregma and lambda, $1 \mathrm{~mm}$ lateral to the midline. The dura mater was kept intact over the cortex. The CCI was performed perpendicular to the brain surface using a PinPoint ${ }^{\mathrm{TM}}$ Precision Cortical Impactor (Cary, NC, USA) with a $3-\mathrm{mm}$ diameter impact tip. The impact velocity is $3 \mathrm{~m} / \mathrm{s}$, the impact duration is $150 \mathrm{~ms}$, and the impact depth is $2 \mathrm{~mm}$. After TBI, the bone flap was immediately replaced, sealed, and the scalp was sutured closed. The animal's core body temperature was maintained at $37 \pm 0.5{ }^{\circ} \mathrm{C}$ with a thermostatically controlled heating pad during surgery. Sham animals were subjected to all aspects of the protocol (surgery, anesthesia, craniotomy, injection, and recovery) except for CCI. Recombinant protein S (PS) $(0.2 \mathrm{mg} / \mathrm{kg})(9489-\mathrm{PS}, \mathrm{R} \& \mathrm{D}$ Systems) was administered via the tail vein at $1 \mathrm{~h}, 1 \mathrm{~d}$, and $2 \mathrm{~d}$ after the CCI $[25,26]$.

\section{Intracerebroventricular (i.c.v.) injection}

Previous studies demonstrated that i.c.v. delivery of siRNA can efficiently silence target gene expression in the brain with a range of 50\%-80\% [27-29]. The i.c.v. administration of siRNA was performed as previously described [30] (Suppl. Fig. 1B). A 1-mm diameter burr hole was drilled into the right side of the skull (1.0 mm lateral to the bregma). Mer siRNA or scrambled siRNA (500 pmol, Thermo Fisher Scientific) mixed with the transfection reagent (Engreen Biosystem Co., Ltd.), for a total volume of $2 \mu \mathrm{l}$, was delivered into the ipsilateral ventricle (1.0 mm lateral of the bregma, $2.5 \mathrm{~mm}$ deep below the skull) $1 \mathrm{~d}$ before TBI induction. The injection was performed at a rate of $0.5 \mu \mathrm{l} / \mathrm{min}$, then the needle stayed in the brain for another $5 \mathrm{~min}$ after injection to avert leakage, and then the burr hole was sealed with bone wax, and the incision was closed with sutures. Mice were placed in individual recovery cages. The i.c.v. injection of siRNA was performed $1 \mathrm{~d}$ before and 10 min after TBI.

\section{Neurobehavioral function assessment}

Modified neurological severity scores (mNSS): The mNSS score was assessed as previously reported [24]. It includes sensory, motor, balance, and reflex tests. The neurological function was graded on a scale of 0-18 (normal score, 0; maximal deficit score, 18) and recorded before TBI, as well as at 1, 3, and $7 \mathrm{~d}$ after TBI. 
Foot-fault test: It was performed to evaluate the motor function similarly to that described previously [30]. Mice were placed on hexagonal grids of different sizes. Mice placed their paws on the wire while moving along the grid. With each weight-bearing step, the paw may fall or slip between the wire. This was recorded as a foot fault. The total number of steps (movement of each forelimb) that the mouse used to cross the grid was counted, and the total number of foot faults for each forelimb was recorded. The percentage of forelimb faults to the total number of steps was calculated.

Rotarod Test: It was performed to test the motor coordination and the limb strength as previously described with the Rota-Rod Treadmills (BW-ZH600, Shanghai Bio-will Co., Ltd.) [30]. Test sessions consist of six trials at a variable speed (an initial velocity of $5 \mathrm{rpm}$ was used for the first $10 \mathrm{~s}$, a linear increase from 5 to $10 \mathrm{rpm}$ for the next $30 \mathrm{~s}$, and a linear increase from 10 to $20 \mathrm{rpm}$ between 40 to $90 \mathrm{~s}$ ). The final score was determined as the mean time that a mouse was able to remain on the rod over six trials.

\section{Brain water content measurement}

Brain water content was measured as previously reported [24]. Briefly, mice were sacrificed $72 \mathrm{~h}$ after TBI, and the brains were obtained without transcardiac perfusion. Tissue samples of injured hemispheres were dissected and weighed on an electric analytic balance to obtain the wet weight and then dried at $100^{\circ} \mathrm{C}$ for $48 \mathrm{~h}$ to obtain the dry weight. Brain water content was calculated using the following formula: brain water content $(\%)=($ wet weight - dry weight)/wet weight $\times 100 \%$.

\section{Contusion volume assessment}

To measure the contusion volume in the ipsilateral cortex $72 \mathrm{~h}$ after TBI, cresyl violet-stained sections were digitized and analyzed by using ImageJ (National Institutes of Health, Bethesda, MD, USA) as previously reported [31]. The volume was computed by adding the injury areas and multiplying with the inter-slice distance $(500 \mu \mathrm{m})$. Hemispheric tissue loss was expressed as a percentage that was calculated by the use of the following formulae: [(contralateral hemispheric volume - ipsilateral hemispheric volume)/(contralateral hemispheric volume $) \times 100 \%$ ].

\section{Quantitative RT-PCR}

Total RNA was isolated from sham and injured brains using the TRIzol reagent (Sigma-Aldrich, St. Louis, MO, USA) according to the manufacturer's instructions. Then, RNA $(1 \mu \mathrm{g})$ from each sample was reverse-transcribed to cDNA by PrimeScript ${ }^{\mathrm{TM}}$ RT reagent kit (Takara Bio Inc, Shiga, Japan). Afterward, RT-PCR was conducted with SYBR ${ }^{\circledR}$ Premix Ex Taq ${ }^{\mathrm{TM}}$ (Takara Bio Inc, Shiga, Japan) on a 7300 Plus Read-Time PCR System (Thermo Fisher Scientific). The cDNA was used as a template in a $20 \mu 1$ reaction volume (10 $\mu 1$ of PCR mix, 5 pmol of forward and reverse primers, $1 \mu \mathrm{cDNA}$ template and proper volume of water). The PCR reaction was performed as follows: Cycling conditions began with an initial DNA denaturation step at $95{ }^{\circ} \mathrm{C}$ for $20 \mathrm{~s}$, followed by 40 cycles at $94{ }^{\circ} \mathrm{C}$ for $15 \mathrm{~s}, 56{ }^{\circ} \mathrm{C}$ for $30 \mathrm{~s}$, and $72{ }^{\circ} \mathrm{C}$ for $25 \mathrm{~s}$. Each sample was examined in triplicate. The threshold cycle (CT) readings were collected, and the relative expression of mRNA of target genes was calculated with the $2^{-\Delta \Delta C T}$ method and was normalized to the glyceraldehyde 3-phosphate dehydrogenase (GAPDH) level in all samples. The expression levels of the mRNA were then reported as fold changes versus sham controls. The sequences of the primer pairs for target genes are as shown below:

\begin{tabular}{|l|l|l|}
\hline Gene & Forward primer sequence $\left(\mathbf{5}^{\prime} \mathbf{-} \mathbf{3}\right.$ ') & Reverse primer sequence (5' - 3') \\
\hline CD16 & TTTGGACACCCAGATGTTTCAG & GTCTTCCTTGAGCACCTGGATC \\
\hline
\end{tabular}




\begin{tabular}{|l|l|l|}
\hline$C D 32$ & AATCCTGCCGTTCCTACTGATC & GTGTCACCGTGTCTTCCTTGAG \\
\hline$i N O S$ & CAAGCACCTTGGAAGAGGAG & AAGGCCAAACACAGCATACC \\
\hline$C D 206$ & CAAGGAAGGTTGGCATTTGT & CCTTTCAGTCCTTTGCAAGC \\
\hline$A r g-1$ & TCACCTGAGCTTTGATGTCG & CTGAAAGGAGCCCTGTCTTG \\
\hline$I L-10$ & CCAAGCCTTATCGGAAATGA & TTTTCACAGGGGAGAAATCG \\
\hline$M e r$ & CCTCTGCTTCGCCACATCTGTATG & GACCAGCCAATCTCATTCCGACAG \\
\hline$S O C S-1$ & CCTCGTCCTCGTCTTCGTCCTC & GAAGGTGCGGAAGTGAGTGTCG \\
\hline$S O C S-3$ & GACCAAGAACCTACGCATCCAGTG & GCACCAGCTTGAGTACACAGTCG \\
\hline$G A P D H$ & TTCAACGGCACAGTCAAGG & CACCAGTGGATGCAGGGAT \\
\hline
\end{tabular}

\section{Western blot}

Western blot was performed as previously described [24]. Briefly, tissue samples from the ipsilateral cortex of sham/TBI mouse brains were homogenized in RIPA buffer $(50 \mathrm{mM}$ Tris- $\mathrm{HCl}$ at $\mathrm{pH} 7.4,150 \mathrm{mM} \mathrm{NaCl}, 1 \%$ Triton X-100, $1 \%$ sodium deoxycholate, $0.1 \%$ SDS, 1 mM EDTA) with protease and phosphatase inhibitors, followed by denaturation at $95{ }^{\circ} \mathrm{C}$ for $10 \mathrm{~min}$. Then the protein samples were separated by SDS-PAGE and transferred onto polyvinylidene fluoride (PVDF) membranes (Millipore). Next, the PVDF membranes were blocked with 5\% bovine serum albumin for $1 \mathrm{~h}$ and incubated with the primary antibodies overnight, including: Anti-Mer antibody (1:1000, Abcam, ab184086), anti-p-Mer antibody (1:500, Abcam, ab192649), anti-STAT1 antibody (1:1000, CST, 14994) anti-p-STAT1 antibody (1: 1000, CST, 9167), anti-SOCS-1 (1:1000, Abcam, ab62584), anti-SOCS-3 (1:1000, Abcam, ab16030), and anti-GAPDH (1:5000, Abcam, ab8245). After that, the PVDF membranes were disposed of with the relevant secondary antibodies (1:5000) for $1 \mathrm{~h}$ at room temperature and observed using the ECL kit chemiluminescence reagents (Millipore, Billerica, MA, USA). The signals of protein bands were detected with the Chemidoc detection system and quantified using Quantity One software (Bio-Rad).

\section{Immunohistochemistry and confocal microscopy analysis}

Mice were anesthetized and transcardially perfused with 0.1 mmol PBS and 4\% paraformaldehyde (PFA) at $3 \mathrm{~d}$ post-TBI. $20 \mu \mathrm{m}$ coronal cryosections were permeabilized and incubated in 5\% Donkey Serum for $1 \mathrm{~h}$ for blocking. Then the brain tissues were incubated in primary antibody overnight at $4{ }^{\circ} \mathrm{C}$. The primary antibody information is as following: anti-Mer antibody (1:400, AF591, R\&D Systems), anti-CD16/32 antibody (1:200, Abcam, ab25235), anti-CD206 antibody (1:400, Abcam ab64693), anti-Iba-1 antibody (1:500, Wako, 019-19741) and (1:500, Abcam, ab5076). After the incubation overnight, the cryosections were incubated with the secondary antibodies (1:500, Jackson Immunoresearch Laboratories) for $1 \mathrm{~h}$ at room temperature. After that, the sections were rinsed with PBS and covered with fluorescence mounting medium with 4',6-diamidino-2-phenylindole (DAPI) (Vector Laboratories, H-1200).

\section{Nissl staining}

Nissl staining was performed according to the manufacturer's instructions. After 10- $\mu \mathrm{m}$ coronal sections had been deparaffinized and rehydrated, the slides were stained in Nissl Staining Solution (C0117, Beyotime) for 5 min at $37{ }^{\circ} \mathrm{C}$. A large cell body, with abundant cytoplasm, and with substantially significant levels of Nissl body represents a normal neuron. However, some other cell forms such as a shrunken cell body, condensed nuclei, reduced or disappearance Nissl body represents a damaged cell. A total of six sections (400-fold field) from each group were used for quantification. 


\section{Fluoro-Jade B (FJB) staining}

The FJB staining was performed as previously described [24]. 20- $\mu \mathrm{m}$ coronal sections were first immersed in a solution containing $1 \%$ sodium hydroxide in $80 \%$ alcohol for $5 \mathrm{~min}$, which followed by $2 \mathrm{~min}$ in $70 \%$ alcohol and $2 \mathrm{~min}$ in distilled water. The slides were then incubated in a solution of $0.06 \%$ potassium permanganate for $10 \mathrm{~min}$, rinsed in distilled water for $2 \mathrm{~min}$ and incubated in a $0.0004 \%$ solution of FJB (Chemicon, Temecula, CA, USA) made in $0.1 \%$ acetic acid for $30 \mathrm{~min}$. The slides were rinsed, air-dried, cleared in xylene for a minute, and coverslipped. All sections were observed and photographed under a fluorescence microscope with blue (450$490 \mathrm{~nm}$ ) excitation light. The total number of FJB-positive cells was expressed as the mean number per 200-fold field in the contusion margin along the cortex.

\section{Statistical analysis}

All data were shown as means \pm standard deviation. For comparison between two groups, the $\mathrm{F}$ test was conducted to determine the similarity in the variances between the groups that are statistically compared, and statistical significance was analyzed by Student's t-test. For multiple comparisons, Bartlett's test for equal variances was used to determine the variances between the multiple groups and one-way analysis of variance (ANOVA) followed by Bonferroni's post hoc test was used to test statistical significance. All analyses were performed using GraphPad Prism 8 software by an investigator blinded to the experimental conditions. A $p$-value $<0.05$ was considered as statistically significant. 


\section{Results}

\section{Dynamic changes in mRNA expression of microglial M1/M2 polarization markers following TBI}

Polarized microglia can be commonly distinguished by their expression profiles of signature surface markers and cytokines/chemokines. To evaluate the microglial activation states following TBI (Suppl. Fig. 2A), RT-PCR was performed using total RNA extractions from the perilesional cortical tissues at $3 \mathrm{~h}, 12 \mathrm{~h}, 1 \mathrm{~d}, 3 \mathrm{~d}$, and $7 \mathrm{~d}$ after TBI or sham-operation (Fig. 1A). Levels of M1-associated markers, including CD16 (Fig. 1B), CD32 (Fig. 1C), and iNOS (Fig. 1D) were gradually increased over time from $12 \mathrm{~h}$ onward and remained elevated for at least $7 \mathrm{~d}$ after injury. The mRNA levels of tested M2 markers, including CD206 (Fig. 1E), Arg-1 (Fig. 1F), and IL-10 (Fig. $1 \mathrm{G})$, began to significantly upregulated at $1 \mathrm{~d}, 12 \mathrm{~h}$, and $1 \mathrm{~d}$ after TBI, respectively, and all decreased at $7 \mathrm{~d}$ postinjury. These results indicated that both M1 and M2 polarized microglia were activated during the acute and subacute phases response after TBI. TBI induces transient upregulation in the M2 phenotype but causes sustained upregulation in the M1 phenotype post-insult, which is consistent with recent reports $[12,13]$.

\section{Expression patterns and cellular localization of Mer following TBI}

Mer plays a critical role in regulating microglial phenotypes and functions under physiological and pathological conditions [21]. We first analyzed the levels of Mer protein and mRNA in the injured cortex at different time points after TBI (Supplemental Fig. 1A). As shown in Fig. 2A and 2B, both Mer protein and mRNA levels were significantly increased $12 \mathrm{~h}$ after TBI, peaked around day 3 and returned to the pre-injury levels around day 7 . We further investigated the cellular localization and expression of Mer in microglia in the ipsilateral cerebral cortex at $3 \mathrm{~d}$ after TBI, using double immunofluorescent staining of Mer and microglial marker Iba-1. As demonstrated in Fig. 2C and 2E, Mer was abundantly expressed in the plasma membrane of microglia in the sham group, and substantially upregulated in the activated microglia with phagocytotic morphology (Fig. 2D and 2E) near the lesion at $3 \mathrm{~d}$ post-TBI.

\section{Inhibition of Mer worsened the functional outcomes after TBI}

Previous studies reported that i.c.v. delivery of siRNA can efficiently silence specific genes in the brain [27-29]. To determine the role of Mer in the pathophysiology of TBI, we administrated Mer siRNA into the ipsilateral ventricle to inhibit its expression in the brain [32] (Suppl. Fig. 2B). Data from the Western blot and RT-PCR demonstrated the knockdown efficacy of Mer siRNA was $>70 \%$ (Fig. 3A and 3B). Besides, we evaluated the functional outcomes following TBI using the mNNS score, foot-fault and rotarod tests performed before and 1, 3, $7 \mathrm{~d}$ after TBI. When compared to the sham group, TBI mice exhibited significantly worse in all neurobehavioral tests (Fig. 3C-E). More importantly, administration of Mer siRNA significantly aggravated the neurobehavioral deficits post-injury when compared with the group received Control siRNA at day 1, 3 and 7 after TBI, based on mNNS scores (Fig. 3C), foot-fault test (Fig. 3D) and rotarod test (Fig. 3E).

To assess the gross pathological changes, we compared brain contusion volume and tissue edema among sham and TBI groups at $3 \mathrm{~d}$ post-injury. As demonstrated, TBI caused significant loss of brain tissue (Fig. 3F) and increased brain edema in the ipsilateral hemisphere (Fig. 3G). Interestingly, although Mer siRNA did not affect the brain contusion volume at day 3 after TBI (Fig. 3F), it significantly elevated brain edema level at $3 \mathrm{~d}$ postinjury, when compared to both the vehicle and Control siRNA group (Fig. 3G). These findings suggested that Mer presence and its upregulation after injury is beneficial for the recovery after TBI.

\section{Mer modulated microglial M1/M2 polarization after TBI}


To investigate the role of Mer in regulating microglial M1/M2 polarization and neuroinflammation following TBI, we evaluated the level of Mer in both M1 and M2 polarized microglia in TBI affected cortical regions. Results from double immunofluorescent staining demonstrated that Mer was expressed in CD16/32-positive M1 cells (Fig. 4A) as well as in CD206-positive M2 cell population (Fig. 4B) in the perilesional area of cortex at $3 \mathrm{~d}$ post-TBI.

To specifically evaluate the effect of Mer siRNA on the M1/M2 polarization state of microglia after TBI, CD16/32 or CD206 was co-labeled with the microglia marker Iba-1 in the cortex surrounding the lesion cavity at $3 \mathrm{~d}$ post-insult, respectively. As demonstrated, the percentage of CD16/32 and Iba-1 double-positive M1 cells was significantly increased in the cortex at day 3 after TBI, which was further elevated in the Mer siRNA group (Fig. 4C and 4D). On the other hand, the percentage of CD206 and Iba-1 double-positive M2 cells was also increased significantly in the ipsilateral cortex at day 3 after TBI when compared with the sham group (Fig. 4E and 4F), but it was significantly decreased following Mer siRNA administration when compared with the TBI + Vehicle or TBI + Control siRNA group (Fig. 4E and 4F). In addition, the ratio between CD16/32 $\mathrm{Iba}-1^{+} \mathrm{M} 1$ cells and CD206 ${ }^{+}$Iba- $^{+}$M2 cells was nearly doubled after Mer siRNA administration (Fig. 4G). Taken together, these findings indicate that Mer plays an important role in regulating microglial M1/M2 polarization following TBI, and inhibition of Mer expression tips the balance of TBI-induced microglial activation towards the proinflammatory M1 phenotype.

\section{Downregulation of Mer aggravated neuronal damage and degeneration following TBI}

To further evaluate the pathological effects of Mer knockdown following TBI, Nissl and FJB staining were employed to access the neuronal damage and degeneration following TBI. As shown, TBI caused a significant decrease in the number of Nissl-positive neurons in the perilesional cortex at $3 \mathrm{~d}$ post-insult (Fig. 5A). Moreover, in vivo knockdown of Mer aggravated neuronal damage following TBI, as demonstrated by the significant decrease in the number of Nissl-positive neurons in the injured cortex in the TBI + Mer siRNA group compared to the TBI + Vehicle or TBI + Control siRNA group (Fig. 5A). Besides, FJB staining showed that TBI induced a significant increase in the number of degenerating neurons in the injured cortex, and Mer siRNA application further increased the number of degenerating neurons in the injured cortex after TBI (Fig. 5B). These data indicated inhibition of Mer expression aggravated neuronal damage and degeneration following TBI.

\section{Inhibition of Mer reduced STAT1 activation and SOCSs expression following TBI}

Previous studies indicated that Mer is a pleiotropic regulator of the innate immune system, and it regulates inflammatory responses through modulating multiple downstream cellular pathways such as STAT1/SOCSs signaling [16,19]. Activation of Mer signaling is associated with phosphorylation of the tyrosine residue 749 in the kinase domain [33]. To clarify the mechanism by which Mer interferes with microglial M1/M2 polarization, we determined the expression of the suppressor of cytokine signaling SOCS-1 and SOCS-3 after TBI, which are key mediators of Mer signaling in regulating inflammatory responses [34,35]. As demonstrated, the levels of phosphorylated Mer (p-Mer) (Fig. 6A) and phosphorylated STAT1 (p-STAT1) (Fig. 6B) were significantly inhibited by Mer siRNA administration on day 3 following TBI. More importantly, the protein and mRNA expression of SOCS-1 (Fig. 6C and 6D) and SOCS-3 (Fig. 6E and 6F) were significantly reduced after TBI in the Mer siRNA treatment group when compared with the Vehicle or Control siRNA group. These findings indicate that Mer signaling is required for activation of STAT1 and SOCSs signaling pathway in the perilesional area of cortex following TBI.

\section{Mer activation alleviated functional deficits following TBI}


To further determine the role of Mer following TBI, administration of recombinant PS (a ligand and activator of Mer) was performed after TBI (Suppl. Fig. 2C and 2D) [36]. Western blot assay revealed PS treatment caused a significant increase of p-STAT1 (Fig. 7A), SOCS-1 (Fig. 7B), and SOCS-3 (Fig. 7C) expression when compared with the vehicle group. Moreover, PS treatment significantly alleviated neurobehavioral deficits at day 3 postinjury, based on the mNNS score (Fig. 7D). More importantly, Mer siRNA administration abolished PS-induced changes in p-STAT1 level (Fig. 7E), and expression levels of SOCS-1 (Fig. 7F) and SOCS-3 (Fig. 7G) in TBI mice. In addition, the mNNS scores showed worsened neurological outcome in mice from the PS + Mer siRNA group when compared with the PS + Control siRNA treatment group. Taken together, these findings suggested that Mer promoted STAT1-mediated upregulation of SOCSs expression and improved functional outcomes following TBI.

\section{Discussion}

Neuroinflammation is conceived as an important manipulable aspect of secondary brain injury following TBI[9]. Here, we demonstrate that Mer upregulation provides neuroprotective effects in a CCI mouse model of TBI via regulating microglial M1/M2 polarization and neuroinflammation (Fig. 8). Mechanistically, Mer mediates activation of the STAT1/SOCSs signaling pathway. Inhibition of Mer markedly decreases microglial M2 polarization while increases M1 polarization, thus aggravates secondary brain damage and functional deficits after TBI.

TBI is an evolving neurological event and it heavily contributes to both acute injuries and chronic neurodegenerative cascades post-insult [37,38]. This disease is challenging to be treated because of its heterogeneous nature and complex pathogenic cascades [39,40]. Of note, microglial activation and neuroinflammation are hallmark features of TBI pathophysiology [7]. As the resident innate immune cells in the CNS, microglia usually respond within minutes toward the sites of damage, where they can even sustain for many years following TBI [9]. Initially, these responses allow them to scavenge debris, promote tissue remodeling and repair, and protect the brain from secondary injury after TBI [41]. However, abnormal activation of microglia can interfere with endogenous repair mechanisms and drives inflammatory damage after TBI [41]. Indeed, microglia have multi-dimensional activation states in CNS diseases depending on different pathophysiological conditions [42]. They can become polarized ranging from the classic M1-like phenotype to an alternative M2-like phenotype after CNS injuries including TBI [7,43]. The M1-like response is presumed to be pro-inflammatory [11], whereas the M2-like phenotype owns anti-inflammatory effects [44]. Multiple molecular pathways, such as STAT, nuclear factor- $\kappa \mathrm{B}(\mathrm{NF}-\mathrm{\kappa B})$ and interferon regulatory factor (IRF), are involved in the regulation of M1/M2 phenotypic transitions [45-47]. Pre-clinical evidence indicated that mixed phenotypes are present in the pathological processes of TBI, which offer opportunities for therapeutic interventions [48]. In the current study, we also found that TBI induces transient upregulation in the M2 phenotype but causes sustained upregulation in the M1 phenotype post-insult, which is consistent with other findings [12,13]. Modulating microglial M1/M2 polarization via regulating those intrinsic molecular switches will facilitate the repair function of microglia and promote neurovascular network restoration after TBI.

Mer, an important member of the TAM family, is predominantly expressed on myeloid-derived hematopoietic cells and functions as a pleiotropic inhibitor of the innate immune response [49]. RNA sequencing data demonstrated that Mer mRNA is highly enriched in microglia in the CNS [50]. In contrast, the mRNA expression levels of the other two family members, namely Axl and Tyro3, are very low in microglia [50]. Moreover, PS and 
growth arrest-specific factor 6 (Gas6), two best-characterized ligands, can bind the Ig1 and Ig2 domains of Mer via their C-terminal regions [51]. Upon ligand stimulation, Mer can be activated and initiate classic ligandinducible dimerization, causing receptor autophosphorylation, recruitment of signaling proteins with SH2 or PTB domains, and activation of downstream pathways. As an example, activation of Mer can induce SOCSs expression and inhibit inflammation through regulating type I interferon receptor (IFNAR)-associated STAT1 pathway [16]. Genetic targeting of Mer can lead to persistent inflammation and tissue damage in a mouse model of acetaminophen-induced acute liver failure [52]. Also, Mer can mediate recognition and subsequent efferocytosis of apoptotic cells to prevent immune responses [53]. In an in vivo murine model of allergic airway inflammation, Mer-mediated apoptotic eosinophil clearance by phagocytes contributes to resolution of allergic airway inflammation [54]. In a murine model of experimental myocardial infarction, deficiency of Mer in a subset of myocardial monocytes suppresses the removal of dying cardiac cells, delays inflammation resolution and reduces systolic performance after myocardial infarction [55]. Proteolytic cleavage of macrophage Mer reduces efferocytosis and inhibits plaque resolution in atherosclerosis [56]. In contrast, in fat-fed low-density lipoprotein receptor-deficient mice whose myeloid cells expressing a cleavage-resistant variant of Mer, higher expression of Mer in macrophage contributes to improved efferocytosis, smaller necrotic cores, thicker fibrous caps, and increased ratios of pro-resolving versus pro-inflammatory lipid mediators in the atherosclerotic lesions [56]. These findings indicate Mer plays a prominent role in the resolution of inflammation through multiple approaches.

Interestingly, the pathophysiological roles of Mer in microglia and CNS has also been explored. Fourgeaud et al. found that Mer regulates multiple features of microglial physiology [21]. It modulates microglial process extension velocity and response to vascular injury [21]. It mediates microglial phagocytosis of apoptotic cells, and adult mice deficient in microglial Mer exhibits marked accumulation of apoptotic cells in the brain neurogenic regions [21]. Induction of Mer expression on plaque-associated immune cells licenses their phagocytic activity and promotes plaque clearance in murine models of $\mathrm{AD}$ [57]. By using microglia isolated from the adult human brain, Healy et al. found that the expression of Mer is downregulated under pro-inflammatory M1 conditions in microglia, and its expression is significantly upregulated after TGF- $\beta$ treatment [23]. Increased Mer expression is associated with increased anti-inflammatory molecule production (i.e., IL-10) and enhanced myelin clearance in both CNS-derived microglia and monocyte-derived macrophages, which permits subsequent tissue repair in multiple sclerosis [23]. These findings demonstrated that Mer is a functional regulator in modulating microglial activity and neuroinflammatory responses in CNS diseases. In our study, we found that Mer is upregulated in the acute phase stage after TBI, and it regulates microglial M1/M2 polarization following TBI. In vivo knockdown of Mer promotes microglial M1 polarization but inhibits M2 polarization, which eventually aggravates secondary brain injury and exacerbates neurobehavioral impairment after TBI.

\section{Conclusions}

In conclusion, our study demonstrated that Mer is upregulated and exerts beneficial effects in the acute stage of TBI via modulating microglial M1/M2 polarization. Inhibition of Mer markedly decreases microglial M2 polarization while increases M1 polarization, which contributes to the aggravation of secondary brain damage and exaggeration of sensorimotor deficits after TBI. 


\section{Abbreviations}

ANOVA: analysis of variance; Arg-1: arginase 1; CCI: controlled cortical impact; CNS: central nervous system diseases; DAPI: 4',6-diamidino-2-phenylindole; FJB: Fluoro-Jade B; Gas6: growth arrest-specific factor 6; IFNAR: type I interferon receptor; IL-1 $\beta$ : interleukin-1 $\beta$; IL-10: interleukin-10; iNOS: inducible nitric oxide synthase; IRF: interferon regulatory factor; Mer: Myeloid-epithelial-reproductive tyrosine kinase; mNSS:

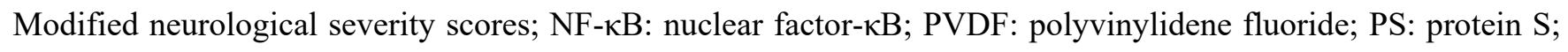
SOCS: suppressor of cytokine signaling; STAT1: signal transducer and activator of transcription 1; TAM: TyroAxl-Mer; TBI: Traumatic brain injury; TGF- $\beta$ : transforming growth factor- $\beta$; TNF- $\alpha$ : tumor necrosis factor- $\alpha$

\section{Acknowledgments}

Not applicable.

\section{Author contributions}

HW and JZ conducted the research and wrote the manuscript; SX, YF, JL, XW, and SM helped to complete the experiment; AS, LS, and YW performed the statistical analysis and edited the manuscript; ZZ edited the manuscript, and JZ guided the entire study, provided the supervision and final check. All the authors read the final version of this paper and approved it.

\section{Funding}

This work was supported by the Young Scientists Fund of the National Natural Science Foundation of China (No. 81701214), the National Natural Science Foundation of China (No. 81870916), the National Key Research and Development Program of China (No. 2017YFC1308500), the Key Program of Science and Technology Development of Zhejiang (No. 2017C03021), and the ZJU Scholarship for Outstanding Doctoral Candidates (No. 2019070).

\section{Availability of data and materials}

The authors confirm that all data underlying the findings are fully available without restriction. All relevant data are within the paper and its supporting information files.

\section{Ethics approval}

Human subjects or samples were not used in this study. All animal experiments were approved by the Institutional Animal Care and Use Committee of Zhejiang University.

\section{Consent for publication}

Not applicable.

\section{Conflicts of interest}

The authors declare that they have no conflict of interest. 


\section{References}

1. Roozenbeek B, Maas AIR, Menon DK. Changing patterns in the epidemiology of traumatic brain injury. Nat Rev Neurol. 2013;9:231-6.

2. Dewan MC, Rattani A, Gupta S, Baticulon RE, Hung Y-C, Punchak M, et al. Estimating the global incidence of traumatic brain injury. J Neurosurg. 2018;1-18.

3. Blennow K, Hardy J, Zetterberg H. The neuropathology and neurobiology of traumatic brain injury. Neuron. 2012;76:886-99.

4. Wilson L, Stewart W, Dams-O'Connor K, Diaz-Arrastia R, Horton L, Menon DK, et al. The chronic and evolving neurological consequences of traumatic brain injury. Lancet Neurol. 2017;16:813-25.

5. Stocchetti N, Zanier ER. Chronic impact of traumatic brain injury on outcome and quality of life: a narrative review. Crit Care Lond Engl. 2016;20:148.

6. Loane DJ, Faden AI. Neuroprotection for traumatic brain injury: translational challenges and emerging therapeutic strategies. Trends Pharmacol Sci. 2010;31:596-604.

7. Simon DW, McGeachy MJ, Bayır H, Clark RSB, Loane DJ, Kochanek PM. The far-reaching scope of neuroinflammation after traumatic brain injury. Nat Rev Neurol. 2017;13:171-91.

8. Witcher KG, Eiferman DS, Godbout JP. Priming the inflammatory pump of the CNS after traumatic brain injury. Trends Neurosci. 2015;38:609-20.

9. Jassam YN, Izzy S, Whalen M, McGavern DB, El Khoury J. Neuroimmunology of Traumatic Brain Injury: Time for a Paradigm Shift. Neuron. 2017;95:1246-65.

10. Pisanu A, Lecca D, Mulas G, Wardas J, Simbula G, Spiga S, et al. Dynamic changes in pro- and antiinflammatory cytokines in microglia after PPAR- $\gamma$ agonist neuroprotective treatment in the MPTPp mouse model of progressive Parkinson's disease. Neurobiol Dis. 2014;71:280-91.

11. Loane DJ, Kumar A. Microglia in the TBI brain: The good, the bad, and the dysregulated. Exp Neurol. 2016;275 Pt 3:316-27.

12. Kumar A, Alvarez-Croda D-M, Stoica BA, Faden AI, Loane DJ. Microglial/Macrophage Polarization Dynamics following Traumatic Brain Injury. J Neurotrauma. 2016;33:1732-50.

13. Wang G, Zhang J, Hu X, Zhang L, Mao L, Jiang X, et al. Microglia/macrophage polarization dynamics in white matter after traumatic brain injury. J Cereb Blood Flow Metab Off J Int Soc Cereb Blood Flow Metab. 2013;33:1864-74.

14. Kumar A, Barrett JP, Alvarez-Croda D-M, Stoica BA, Faden AI, Loane DJ. NOX2 drives M1-like microglial/macrophage activation and neurodegeneration following experimental traumatic brain injury. Brain Behav Immun. 2016;58:291-309.

15. Wang G, Shi Y, Jiang X, Leak RK, Hu X, Wu Y, et al. HDAC inhibition prevents white matter injury by modulating microglia/macrophage polarization through the GSK3 $\beta /$ PTEN/Akt axis. Proc Natl Acad Sci U S A. 2015;112:2853-8. 
16. Rothlin CV, Carrera-Silva EA, Bosurgi L, Ghosh S. TAM receptor signaling in immune homeostasis. Annu Rev Immunol. 2015;33:355-91.

17. Cai B, Kasikara C, Doran AC, Ramakrishnan R, Birge RB, Tabas I. MerTK signaling in macrophages promotes the synthesis of inflammation resolution mediators by suppressing CaMKII activity. Sci Signal. $2018 ; 11$.

18. Choi J-Y, Seo JY, Yoon Y-S, Lee Y-J, Kim H-S, Kang JL. Mer signaling increases the abundance of the transcription factor LXR to promote the resolution of acute sterile inflammation. Sci Signal. 2015;8:ra21.

19. Lee Y-J, Han J-Y, Byun J, Park H-J, Park E-M, Chong YH, et al. Inhibiting Mer receptor tyrosine kinase suppresses STAT1, SOCS1/3, and NF- $\mathrm{BB}$ activation and enhances inflammatory responses in lipopolysaccharideinduced acute lung injury. J Leukoc Biol. 2012;91:921-32.

20. Cai B, Thorp EB, Doran AC, Subramanian M, Sansbury BE, Lin C-S, et al. MerTK cleavage limits proresolving mediator biosynthesis and exacerbates tissue inflammation. Proc Natl Acad Sci U S A. 2016;113:6526-31.

21. Fourgeaud L, Través PG, Tufail Y, Leal-Bailey H, Lew ED, Burrola PG, et al. TAM receptors regulate multiple features of microglial physiology. Nature. 2016;532:240-4.

22. Ji R, Tian S, Lu HJ, Lu Q, Zheng Y, Wang X, et al. TAM receptors affect adult brain neurogenesis by negative regulation of microglial cell activation. J Immunol Baltim Md 1950. 2013;191:6165-77.

23. Healy LM, Perron G, Won S-Y, Michell-Robinson MA, Rezk A, Ludwin SK, et al. MerTK Is a Functional Regulator of Myelin Phagocytosis by Human Myeloid Cells. J Immunol Baltim Md 1950. 2016;196:3375-84.

24. Wu H, Shao A, Zhao M, Chen S, Yu J, Zhou J, et al. Melatonin attenuates neuronal apoptosis through upregulation of $\mathrm{K}(+)-\mathrm{Cl}(-)$ cotransporter $\mathrm{KCC} 2$ expression following traumatic brain injury in rats. J Pineal Res. 2016;61:241-50.

25. Zhu D, Wang Y, Singh I, Bell RD, Deane R, Zhong Z, et al. Protein S controls hypoxic/ischemic blood-brain barrier disruption through the TAM receptor Tyro3 and sphingosine 1-phosphate receptor. Blood.

2010;115:4963-72.

26. Liu D, Guo H, Griffin JH, Fernández JA, Zlokovic BV. Protein S confers neuronal protection during ischemic/hypoxic injury in mice. Circulation. 2003;107:1791-6.

27. Nikolakopoulou AM, Montagne A, Kisler K, Dai Z, Wang Y, Huuskonen MT, et al. Pericyte loss leads to circulatory failure and pleiotrophin depletion causing neuron loss. Nat Neurosci. 2019;22:1089-98.

28. Godinho BMDC, Henninger N, Bouley J, Alterman JF, Haraszti RA, Gilbert JW, et al. Transvascular Delivery of Hydrophobically Modified siRNAs: Gene Silencing in the Rat Brain upon Disruption of the BloodBrain Barrier. Mol Ther J Am Soc Gene Ther. 2018;26:2580-91.

29. Ma Q, Chen S, Hu Q, Feng H, Zhang JH, Tang J. NLRP3 inflammasome contributes to inflammation after intracerebral hemorrhage. Ann Neurol. 2014;75:209-19.

30. Zheng J, Sun Z, Liang F, Xu W, Lu J, Shi L, et al. AdipoRon Attenuates Neuroinflammation After Intracerebral Hemorrhage Through AdipoR1-AMPK Pathway. Neuroscience. 2019;412:116-30. 
31. Batsaikhan B, Wang J-Y, Scerba MT, Tweedie D, Greig NH, Miller JP, et al. Post-Injury Neuroprotective Effects of the Thalidomide Analog 3,6'-Dithiothalidomide on Traumatic Brain Injury. Int J Mol Sci. 2019;20.

32. Tong L-S, Shao A-W, Ou Y-B, Guo Z-N, Manaenko A, Dixon BJ, et al. Recombinant Gas6 augments Axl and facilitates immune restoration in an intracerebral hemorrhage mouse model. J Cereb Blood Flow Metab Off J Int Soc Cereb Blood Flow Metab. 2017;37:1971-81.

33. Ling L, Templeton D, Kung HJ. Identification of the major autophosphorylation sites of Nyk/Mer, an NCAMrelated receptor tyrosine kinase. J Biol Chem. 1996;271:18355-62.

34. van den Brand BT, Abdollahi-Roodsaz S, Vermeij EA, Bennink MB, Arntz OJ, Rothlin CV, et al. Therapeutic efficacy of Tyro3, Axl, and Mer tyrosine kinase agonists in collagen-induced arthritis. Arthritis Rheum.

2013;65:671-80.

35. Zhen Y, Finkelman FD, Shao W-H. Mechanism of Mer receptor tyrosine kinase inhibition of glomerular endothelial cell inflammation. J Leukoc Biol. 2018;103:709-17.

36. Liao D, Wang X, Li M, Lin PH, Yao Q, Chen C. Human protein S inhibits the uptake of AcLDL and expression of SR-A through Mer receptor tyrosine kinase in human macrophages. Blood. 2009;113:165-74.

37. Sivanandam TM, Thakur MK. Traumatic brain injury: a risk factor for Alzheimer's disease. Neurosci Biobehav Rev. 2012;36:1376-81.

38. Smith DH, Johnson VE, Trojanowski JQ, Stewart W. Chronic traumatic encephalopathy - confusion and controversies. Nat Rev Neurol. 2019;15:179-83.

39. Cruz-Haces M, Tang J, Acosta G, Fernandez J, Shi R. Pathological correlations between traumatic brain injury and chronic neurodegenerative diseases. Transl Neurodegener. 2017;6:20.

40. Puntambekar SS, Saber M, Lamb BT, Kokiko-Cochran ON. Cellular players that shape evolving pathology and neurodegeneration following traumatic brain injury. Brain Behav Immun. 2018;71:9-17.

41. Morganti-Kossmann MC, Semple BD, Hellewell SC, Bye N, Ziebell JM. The complexity of neuroinflammation consequent to traumatic brain injury: from research evidence to potential treatments. Acta Neuropathol (Berl). 2019;137:731-55.

42. Li Q, Barres BA. Microglia and macrophages in brain homeostasis and disease. Nat Rev Immunol. 2018;18:225-42.

43. Hu X, Leak RK, Shi Y, Suenaga J, Gao Y, Zheng P, et al. Microglial and macrophage polarization-new prospects for brain repair. Nat Rev Neurol. 2015;11:56-64.

44. Cherry JD, Olschowka JA, O'Banion MK. Neuroinflammation and M2 microglia: the good, the bad, and the inflamed. J Neuroinflammation. 2014;11:98.

45. Kobayashi K, Imagama S, Ohgomori T, Hirano K, Uchimura K, Sakamoto K, et al. Minocycline selectively inhibits M1 polarization of microglia. Cell Death Dis. 2013;4:e525.

46. Qin H, Yeh W-I, De Sarno P, Holdbrooks AT, Liu Y, Muldowney MT, et al. Signal transducer and activator of transcription-3/suppressor of cytokine signaling-3 (STAT3/SOCS3) axis in myeloid cells regulates neuroinflammation. Proc Natl Acad Sci U S A. 2012;109:5004-9. 
47. Tanaka T, Murakami K, Bando Y, Yoshida S. Interferon regulatory factor 7 participates in the M1-like microglial polarization switch. Glia. 2015;63:595-610.

48. Xu H, Wang Z, Li J, Wu H, Peng Y, Fan L, et al. The Polarization States of Microglia in TBI: A New Paradigm for Pharmacological Intervention. Neural Plast. 2017;2017:5405104.

49. Zhang B, Lu H, Jiang A, Wu H, Fang L, Lv Y. MerTK Downregulates Lipopolysaccharide-Induced Inflammation Through SOCS1 Protein but Does Not Affect Phagocytosis of Escherichia coli in Macrophages. Inflammation. 2019;42:113-23.

50. Bennett ML, Bennett FC, Liddelow SA, Ajami B, Zamanian JL, Fernhoff NB, et al. New tools for studying microglia in the mouse and human CNS. Proc Natl Acad Sci U S A. 2016;113:E1738-1746.

51. Lemke G. Biology of the TAM receptors. Cold Spring Harb Perspect Biol. 2013;5:a009076.

52. Triantafyllou E, Pop OT, Possamai LA, Wilhelm A, Liaskou E, Singanayagam A, et al. MerTK expressing hepatic macrophages promote the resolution of inflammation in acute liver failure. Gut. 2018;67:333-47.

53. DeBerge M, Yeap XY, Dehn S, Zhang S, Grigoryeva L, Misener S, et al. MerTK Cleavage on Resident Cardiac Macrophages Compromises Repair After Myocardial Ischemia Reperfusion Injury. Circ Res. 2017;121:930-40.

54. Felton JM, Lucas CD, Dorward DA, Duffin R, Kipari T, Vermeren S, et al. Mer-mediated eosinophil efferocytosis regulates resolution of allergic airway inflammation. J Allergy Clin Immunol. 2018;142:18841893.e6.

55. Wan E, Yeap XY, Dehn S, Terry R, Novak M, Zhang S, et al. Enhanced efferocytosis of apoptotic cardiomyocytes through myeloid-epithelial-reproductive tyrosine kinase links acute inflammation resolution to cardiac repair after infarction. Circ Res. 2013;113:1004-12.

56. Cai B, Thorp EB, Doran AC, Sansbury BE, Daemen MJAP, Dorweiler B, et al. MerTK receptor cleavage promotes plaque necrosis and defective resolution in atherosclerosis. J Clin Invest. 2017;127:564-8.

57. Savage JC, Jay T, Goduni E, Quigley C, Mariani MM, Malm T, et al. Nuclear receptors license phagocytosis by trem2+ myeloid cells in mouse models of Alzheimer's disease. J Neurosci Off J Soc Neurosci. 2015;35:653243. 
Figures and legends

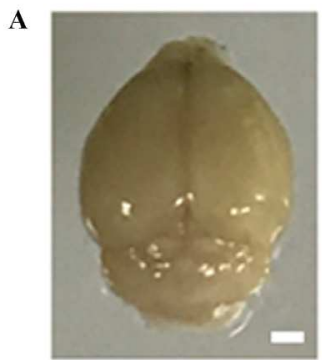

Sham

B

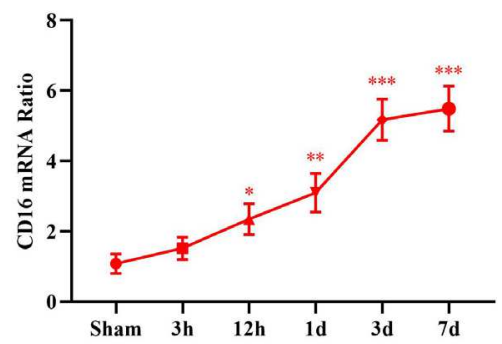

$\mathbf{E}$

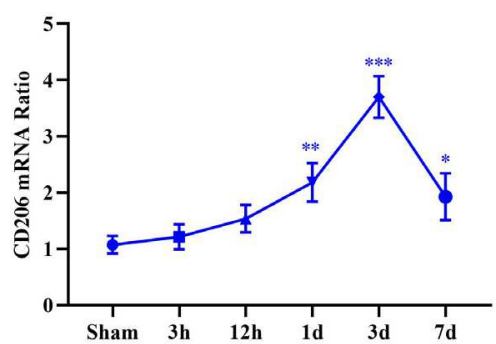

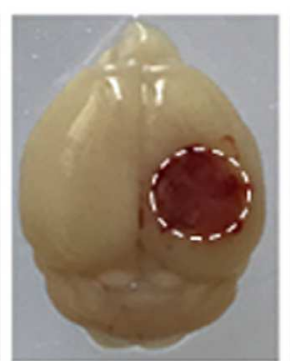

$12 \mathrm{~h}$

C

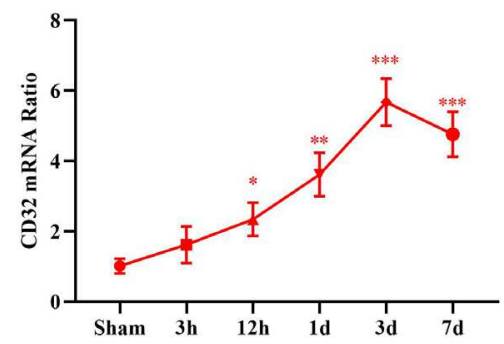

$\mathbf{F}$

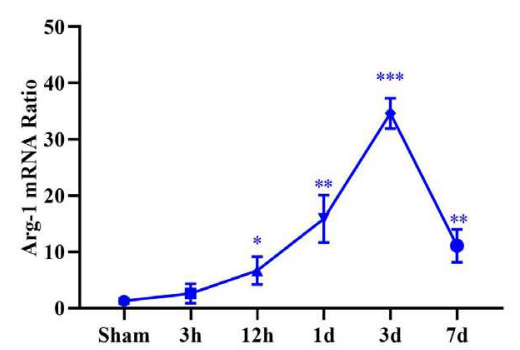

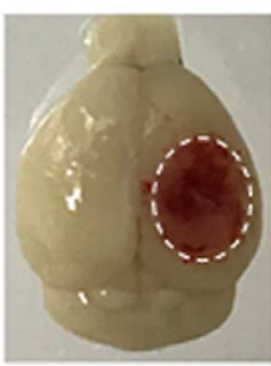

3d

D

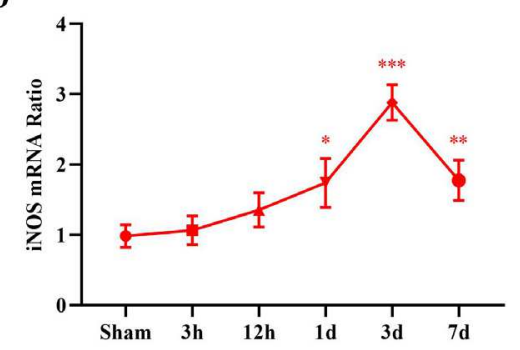

G

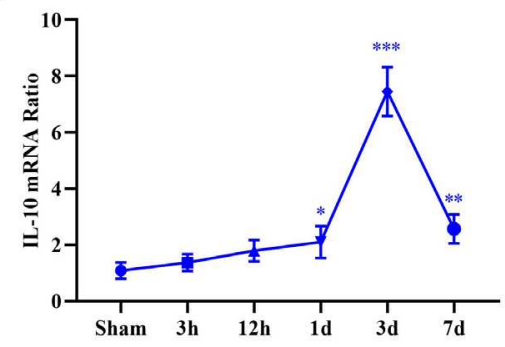

Figure 1. Dynamic changes in mRNA expression of microglial M1 and M2 phenotypic markers following TBI. (A) Representative photographs of whole brains in the sham and different traumatic brain injury (TBI) groups (at $3 \mathrm{~h}, 12 \mathrm{~h}, 1 \mathrm{~d}, 3 \mathrm{~d}$, and $7 \mathrm{~d}$ post-insult, respectively). Scale bar $=1 \mathrm{~mm}$. Quantitative ReverseTranscription Polymerase Chain Reaction (RT-PCR) was used to assess the mRNA expression levels of microglial M1 and M2 phenotypic markers in the injured cortex at $3 \mathrm{~h}, 12 \mathrm{~h}, 1 \mathrm{~d}, 3 \mathrm{~d}$, and $7 \mathrm{~d}$ after TBI or the equivalent area of the sham-operated brains. (B-D) Expressions of mRNA of M1 phenotypic markers, including CD16 (B), CD32 (C), and iNOS (D), were gradually increased over time from $12 \mathrm{~h}$ onward and remained elevated for at least $7 \mathrm{~d}$ after injury. (E-G) Expressions of mRNA of M2 phenotypic markers, including CD206 (E), Arg-1 (F), and IL-10 (G), were significantly up-regulated at $1 \mathrm{~d}, 12 \mathrm{~h}$, and $1 \mathrm{~d}$ after TBI, respectively, and all decreased at $7 \mathrm{~d}$ post-injury. Data are expressed as fold change compared to sham-operated controls. $\mathrm{n}=6$ mice per group. *, $p<0.05$; $^{*}, p<0.01 ;^{* *}, p<0.001$. one-way ANOVA followed by Bonferroni's post-hoc tests. 

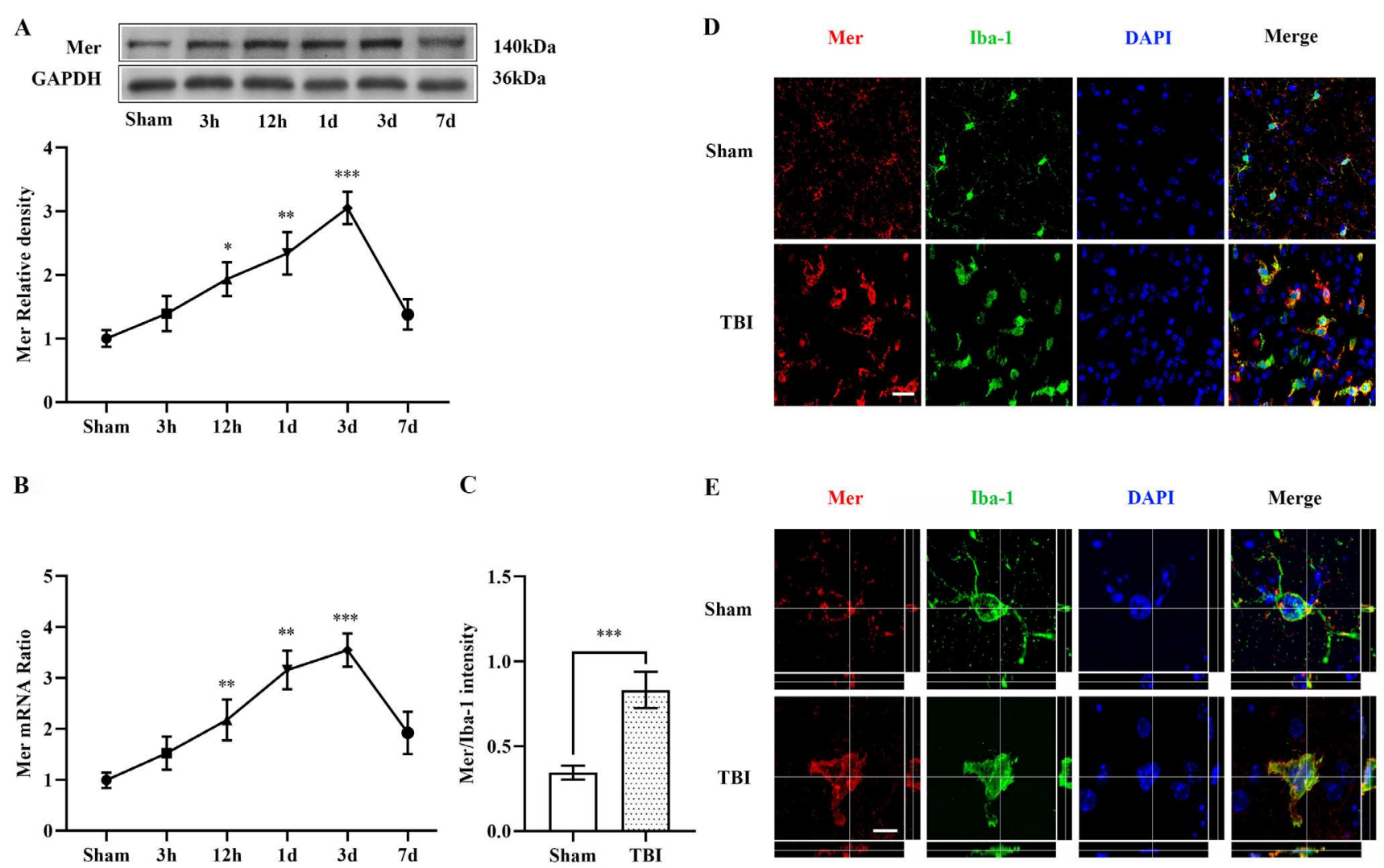

Figure 2. Expression patterns and cellular localization of Mer following TBI. (A) Representative immunoblots and quantification showing the expression level of Mer protein in the injured cortex at $3 \mathrm{~h}, 12 \mathrm{~h}, 1 \mathrm{~d}$, $3 \mathrm{~d}$, and $7 \mathrm{~d}$ after TBI or the equivalent area of the sham-operated brains. Data are expressed as fold change compared to sham-operated controls. $\mathrm{n}=6$ mice per group. (B) Quantitative RT-PCR was used to assess the mRNA expression level of Mer in the injured cortex at $3 \mathrm{~h}, 12 \mathrm{~h}, 1 \mathrm{~d}, 3 \mathrm{~d}$, and $7 \mathrm{~d}$ after TBI or the equivalent area of the sham-operated brains. Data are expressed as fold change compared to sham-operated controls. $\mathrm{n}=6$ mice per group. Double immunofluorescent staining of Mer with the microglial marker Iba-1 in the ipsilateral cerebral cortex was performed at $3 \mathrm{~d}$ post-TBI or sham-operation. (C) Representative confocal images from the ipsilateral cortex showing Mer was abundantly expressed in the plasma membrane of microglia and substantially upregulated in activated microglia following TBI. Scale bar $=15 \mu \mathrm{m}$. (D) Fluorescence intensity quantification of Mer expression in activated microglia in the impacted cortical area 3 days after TBI, compared to that in resting microglia from sham-operated brains. $\mathrm{n}=6$ mice per group. (E) Confocal microscopy analysis at a single-cell resolution showing Mer expression was substantially upregulated in the activated microglia with phagocytotic morphology at $3 \mathrm{~d}$ post-TBI. Orthogonal views demonstrated the colocalization of Mer and Iba-1 in microglia. Scale bar $=5 \mu \mathrm{m}$. In A-B, data are presented as mean $\pm \mathrm{SD} ;{ }^{*}, p<0.05$; $^{* *}, p<0.01 ; * *, p<0.001$. one-way ANOVA followed by Bonferroni's post-hoc tests. In $\mathbf{D}$, data are presented as Mean $\pm \mathrm{SD}$; ***, $p<0.001$ by Student's t-test. 

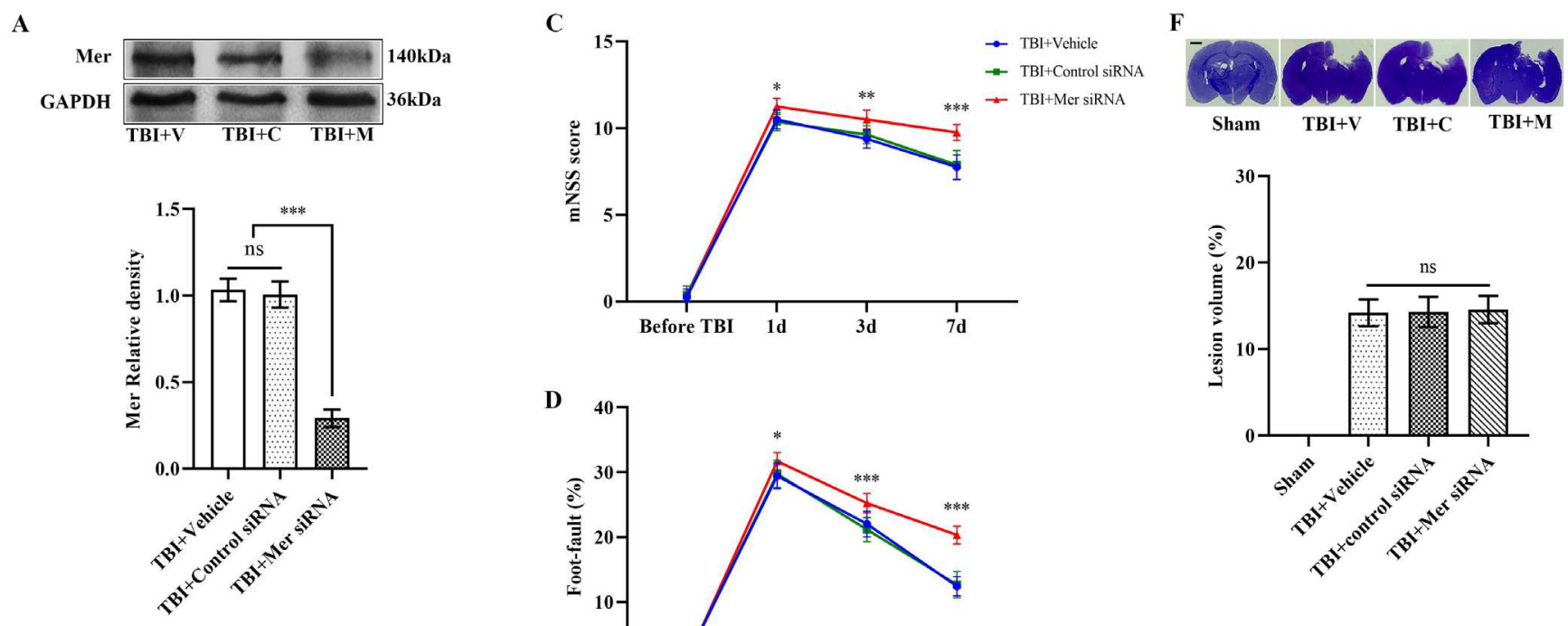

B
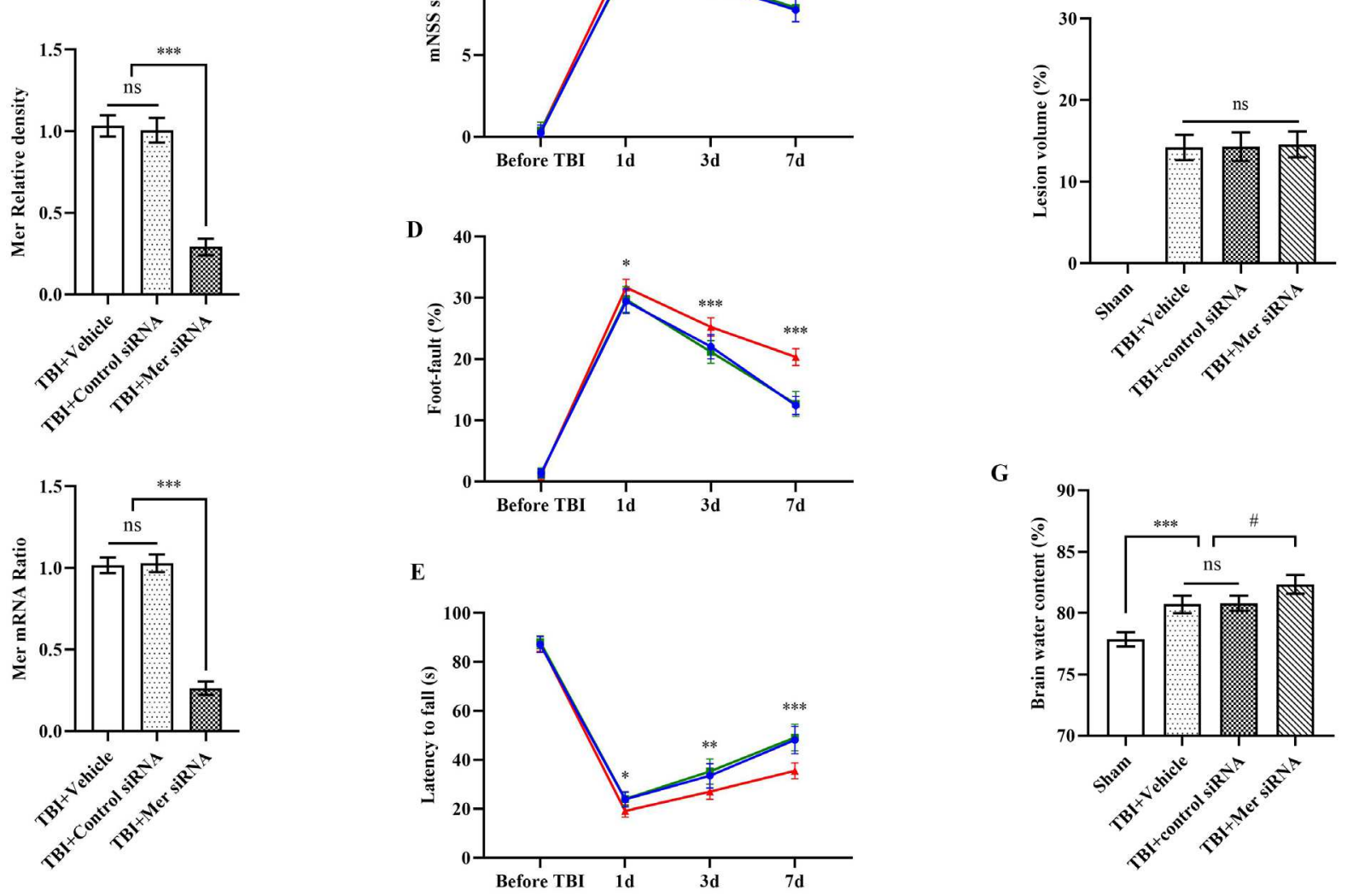

Figure 3. In vivo knockdown of Mer worsened the functional outcomes after TBI. (A) Western blot was used to assess the knockdown efficacy of Mer siRNA. Representative immunoblots and quantification showing Mer siRNA inhibited the expression of Mer protein in the injured cortex at $3 \mathrm{~d}$ post-TBI. TBI + Vehicle: TBI $+\mathrm{V}$, TBI + Control siRNA: TBI + C, TBI + Mer siRNA: TBI + M. GAPDH: loading control. Data are expressed as fold change compared to the TBI + V group; $n=6$ mice per group. (B) Quantitative RT-PCR analysis also showing Mer siRNA significantly inhibited the expression of Mer mRNA in the injured cortex at $3 \mathrm{~d}$ post-TBI. Data are expressed as fold change compared to the TBI $+\mathrm{V}$ group; $\mathrm{n}=6$ mice per group. (C-E) Modified neurological severity scores (mNSS) (C), foot-fault test (D), and rotarod test (E) performed before and 1, 3, and $7 \mathrm{~d}$ after TBI. $\mathrm{n}=8$ mice per group. (F) Quantification of TBI-induced lesion volume at $3 \mathrm{~d}$ post-insult. $\mathrm{n}=6$ mice per group. Scale bar $=1 \mathrm{~mm}$. (G) Cerebral edema was measured by brain water content. Mer siRNA significantly elevated brain edema level at $3 \mathrm{~d}$ post-injury, when compared to both the Vehicle and Control siRNA group. $\mathrm{n}=8$ mice per group. In A-G, data are presented as mean $\pm \mathrm{SD} ; *, \#, p<0.05$; **, $p<0.01$, ***, $p<0.001$, ns, nonsignificant, $p>0.05$. one-way ANOVA followed by Bonferroni's post-hoc tests. 

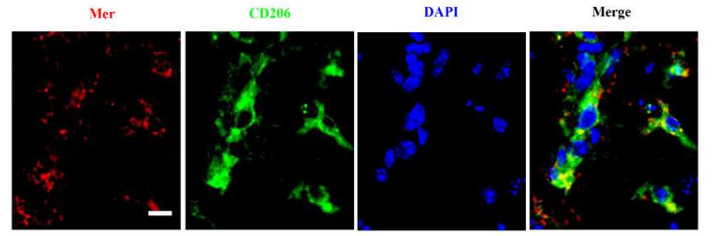

C
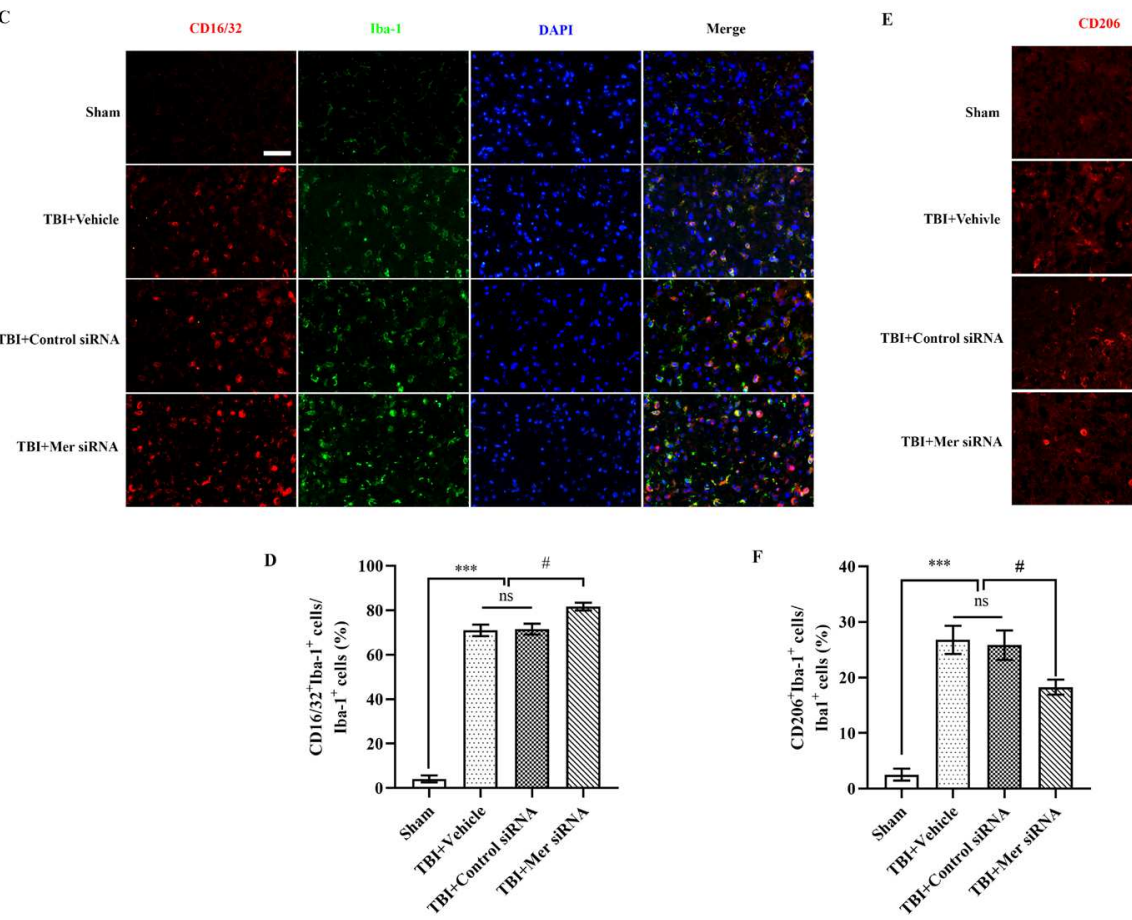

Mer
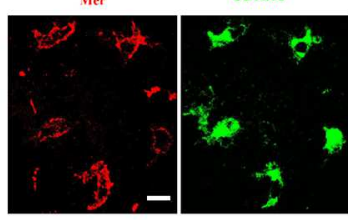

1ba-1

DAPI

DAPI Merge

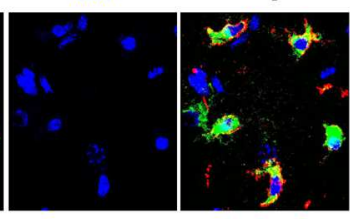

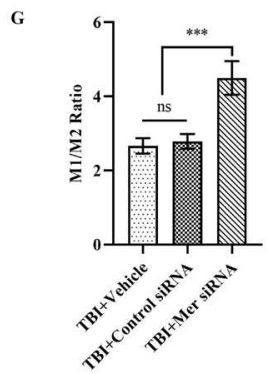

Figure 4. Mer modulated microglial M1/M2 polarization after TBI. (A) Representative images of immunofluorescent staining for Mer (red), CD16/32 (green) and DAPI (blue) showing Mer was expressed in CD16/32-positive cells in the perilesional area of cortex at $3 \mathrm{~d}$ post-TBI. Scale bar $=20 \mu \mathrm{m}$. (B) Representative images of immunofluorescent staining for Mer (red), CD206 (green) and DAPI (blue) showing Mer was expressed in CD206-positive cells in the perilesional area of cortex at $3 \mathrm{~d}$ post-TBI. Scale bar $=20 \mu \mathrm{m}$. (C) Representative images of immunofluorescent staining for CD16/32 (red), Iba-1 (green) and DAPI (blue) in the ipsilateral cortex at $3 \mathrm{~d}$ post-TBI. Scale bar $=50 \mu \mathrm{m}$. (D) Quantification showing the percentage of CD16/32 and Iba-1 double-positive cells was significantly increased in the cortex at day 3 after TBI, which was further elevated in the Mer siRNA group. $\mathrm{n}=6$ mice per group. (E) Representative images of immunofluorescent staining for Mer (red), CD206 (green) and DAPI (blue) in the ipsilateral cortex at $3 \mathrm{~d}$ post-TBI. Scale bar $=50 \mu \mathrm{m}$. (F) Quantification showing the percentage of CD206 and Iba-1 double-positive cells increased significantly in the ipsilateral cortex at day 3 after TBI, however, it significantly decreased following Mer siRNA administration. (G) The ratio between $\mathrm{CD} 16 / 32^{+} \mathrm{Iba}-1^{+} \mathrm{M} 1$ cells and $\mathrm{CD} 206^{+} \mathrm{Iba}-1^{+} \mathrm{M} 2$ cells nearly doubled after Mer siRNA administration. In $\mathrm{D}, \mathrm{F}$, and $\mathrm{G}$, data are presented as mean $\pm \mathrm{SD} ; \#, p<0.05$; ***, $p<0.001$; ns, non-significant, $p>0.05$. one-way ANOVA followed by Bonferroni's post-hoc tests. 
A

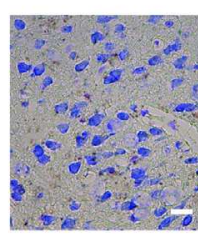

Sham

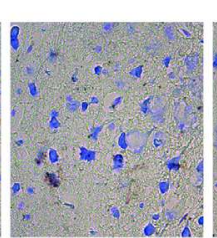

$\mathbf{T B I}+\mathbf{V}$

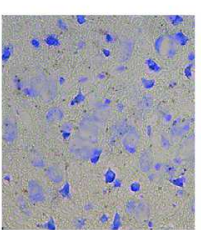

$\mathrm{TBI}+\mathrm{C}$

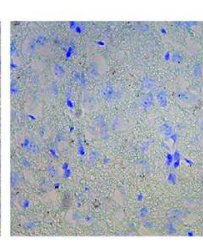

TBI+M

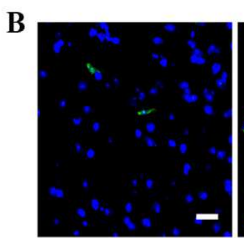

Sham

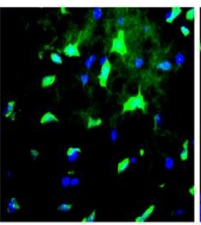

TBI+V

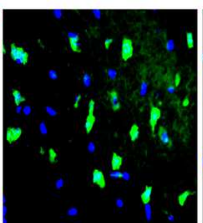

$\mathrm{TBI}+\mathrm{C}$

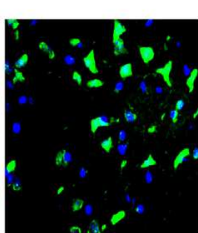

TBI+M
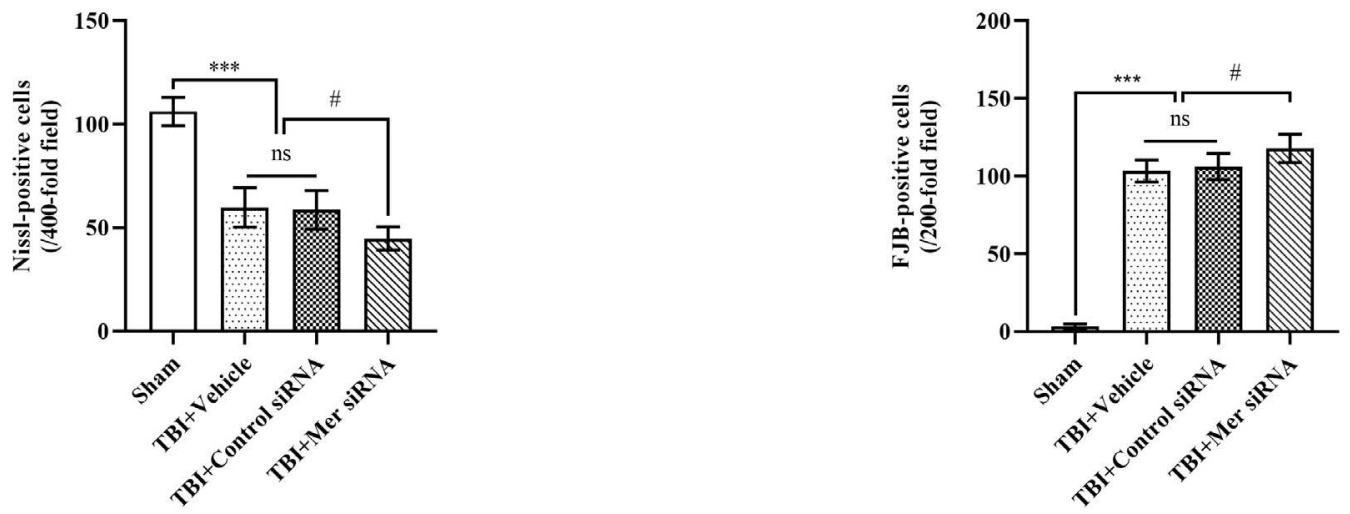

Figure 5. Inhibition of Mer aggravated neuronal damage and degeneration following TBI. (A) Representative images of Nissl staining in the ipsilateral cortex from the sham, TBI + Vehicle (TBI + V), TBI + Control siRNA $(\mathrm{TBI}+\mathrm{C}$ ) and TBI + Mer siRNA (TBI $+\mathrm{M})$ groups, respectively. Quantification analysis showing TBI caused a significant decrease in the number of Nissl-positive cells in the ipsilateral cortex at $3 \mathrm{~d}$ post-TBI, and Mer siRNA application further decreased the number of Nissl-positive cells in the injured cortex after TBI. $n=6$ mice per group. Scale bar $=20 \mu \mathrm{m}$. (B) Representative images of Fluoro-Jade B (FJB) staining in the ipsilateral cortex from the sham, TBI $+\mathrm{V}, \mathrm{TBI}+\mathrm{C}$, and TBI $+\mathrm{M}$ groups, respectively. Quantification analysis showing TBI caused a significant increase in the number of FJB-positive cells in the ipsilateral cortex at $3 \mathrm{~d}$ postTBI, and Mer siRNA application further increased the number of FJB-positive cells in the injured cortex after TBI. $\mathrm{n}=6$ mice per group. Scale bar $=20 \mu \mathrm{m}$. In A-B, data are presented as Mean $\pm \mathrm{SD} ; \#, p<0.05 ; * * *, p<0.001$; ns, non-significant, $p>0.05$. one-way ANOVA followed by Bonferroni's post-hoc tests. 

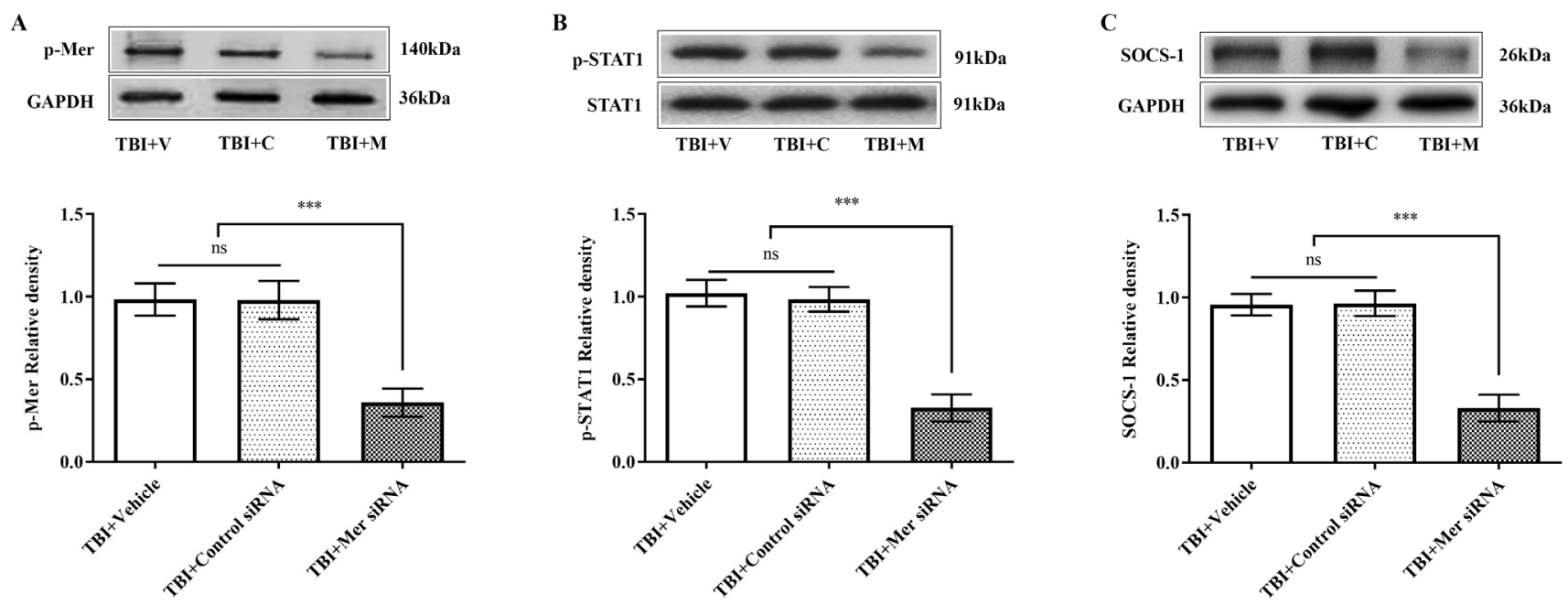

D

E
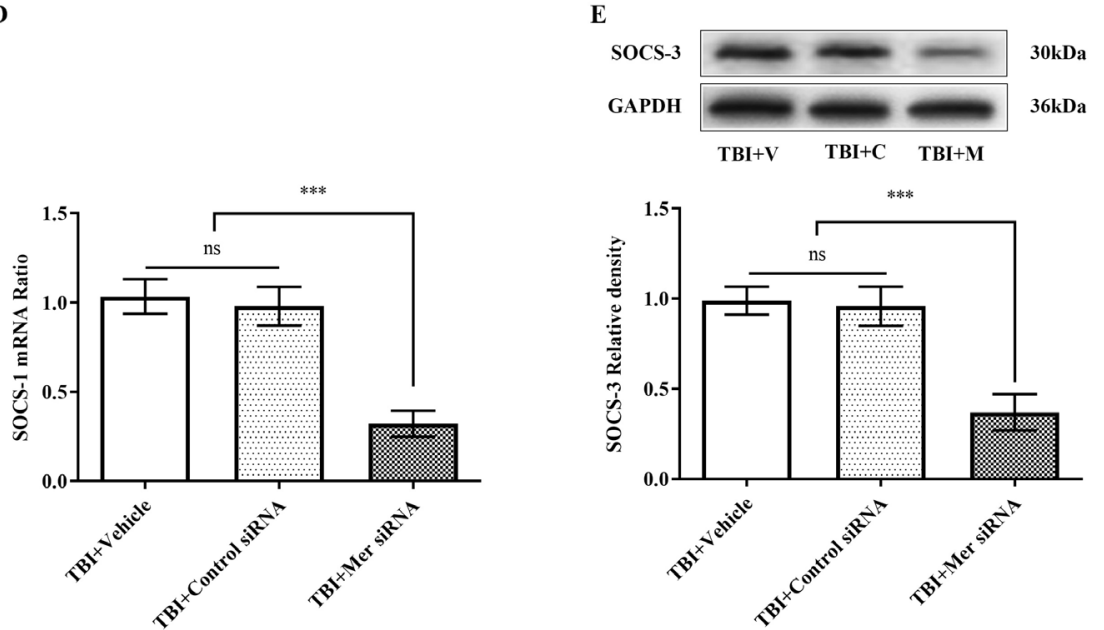

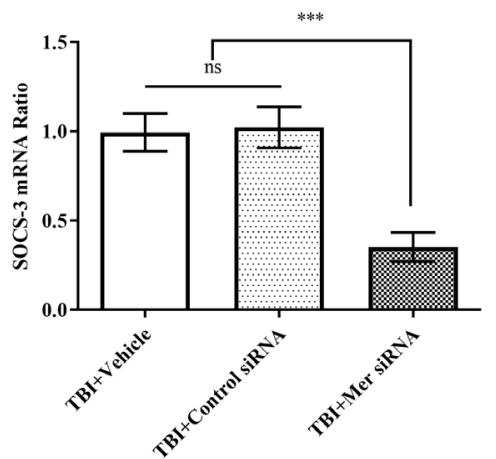

Figure 6. Inhibition of Mer reduced STAT1 activation and SOCSs expression following TBI. (A, B, C, E) Representative immunoblots and quantification showing the expression of phosphorylated Mer (p-Mer) (A), phosphorylated STAT1 (p-STAT1) (B), SOCS-1 (C) and SOCS-3 (E) was significantly inhibited by Mer siRNA administration on day 3 following TBI. TBI + Vehicle: TBI + V, TBI + Control siRNA: TBI + C; TBI + Mer siRNA: TBI + M. GAPDH: loading control. Data are expressed as fold change compared to the TBI + V group; $n$ $=6$ mice per group. (D, F) Quantitative RT-PCR analysis showing Mer siRNA application significantly inhibited the mRNA expression of SOCS-1 (D) and SOCS-3 (F) in the injured cortex at $3 \mathrm{~d}$ post-TBI. Data are expressed as fold change compared to the TBI $+\mathrm{V}$ group; $\mathrm{n}=6$ mice per group. In $\mathbf{A}-\mathbf{F}$, data are presented as Mean $\pm \mathrm{SD}$; $\mathrm{ns}$, non-significant, $p>0.05 ; * * * p<0.001$. one-way ANOVA followed by Bonferroni's post-hoc tests. 

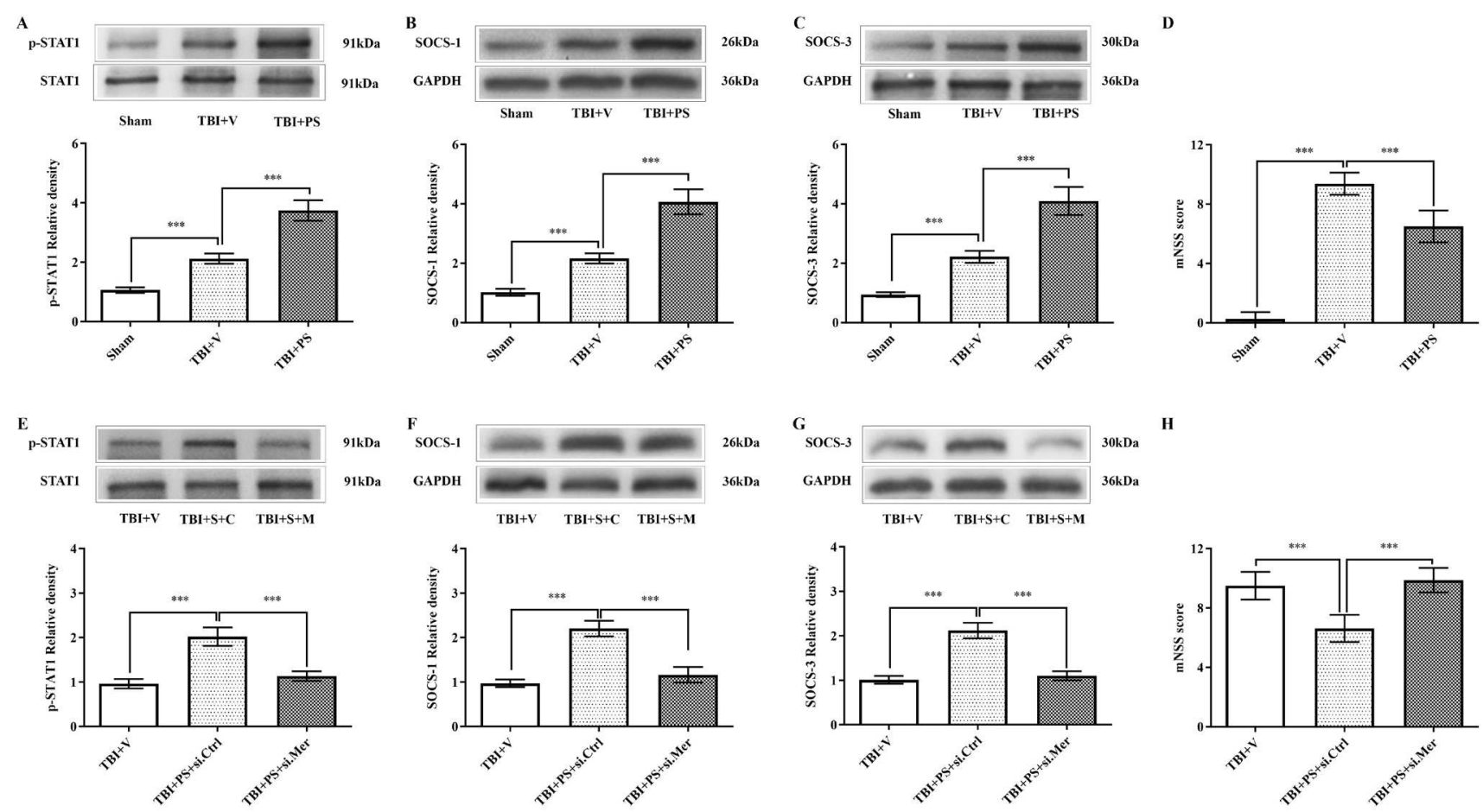

Figure 7. Mer activation alleviated functional deficits following TBI. (A-C) Representative immunoblots and quantification showing protein expression of p-STAT1 (A), SOCS-1 (B), and SOCS-3 (C) in the injured cortex at 3 d post-TBI; TBI + Vehicle: TBI + V, TBI + recombinant protein S: TBI + PS. GAPDH: loading control. Data are expressed as fold change compared to the sham group; $n=6$ mice per group. (D) Modified neurological severity scores (mNSS) performed at $3 \mathrm{~d}$ after TBI. $\mathrm{n}=8$ mice per group. (E-G) Representative immunoblots and quantification showing protein expression of p-STAT1 (E), SOCS-1 (F), and SOCS-3 (G) in the injured cortex at $3 \mathrm{~d}$ after TBI; TBI + Vehicle: TBI + V, TBI + PS + Control siRNA (si.Ctrl): TBI + S + C, TBI + S + Mer siRNA (si.Mer): TBI $+\mathrm{S}+\mathrm{M}$. GAPDH: loading control. Data are expressed as fold change compared to the TBI $+\mathrm{V}$ group; $n=6$ mice per group. (H) The mNSS performed at $3 \mathrm{~d}$ after TBI. $\mathrm{n}=8$ mice per group. In A-H, data are presented as Mean $\pm \mathrm{SD} ; * *, p<0.001$. one-way ANOVA followed by Bonferroni's post-hoc tests. 


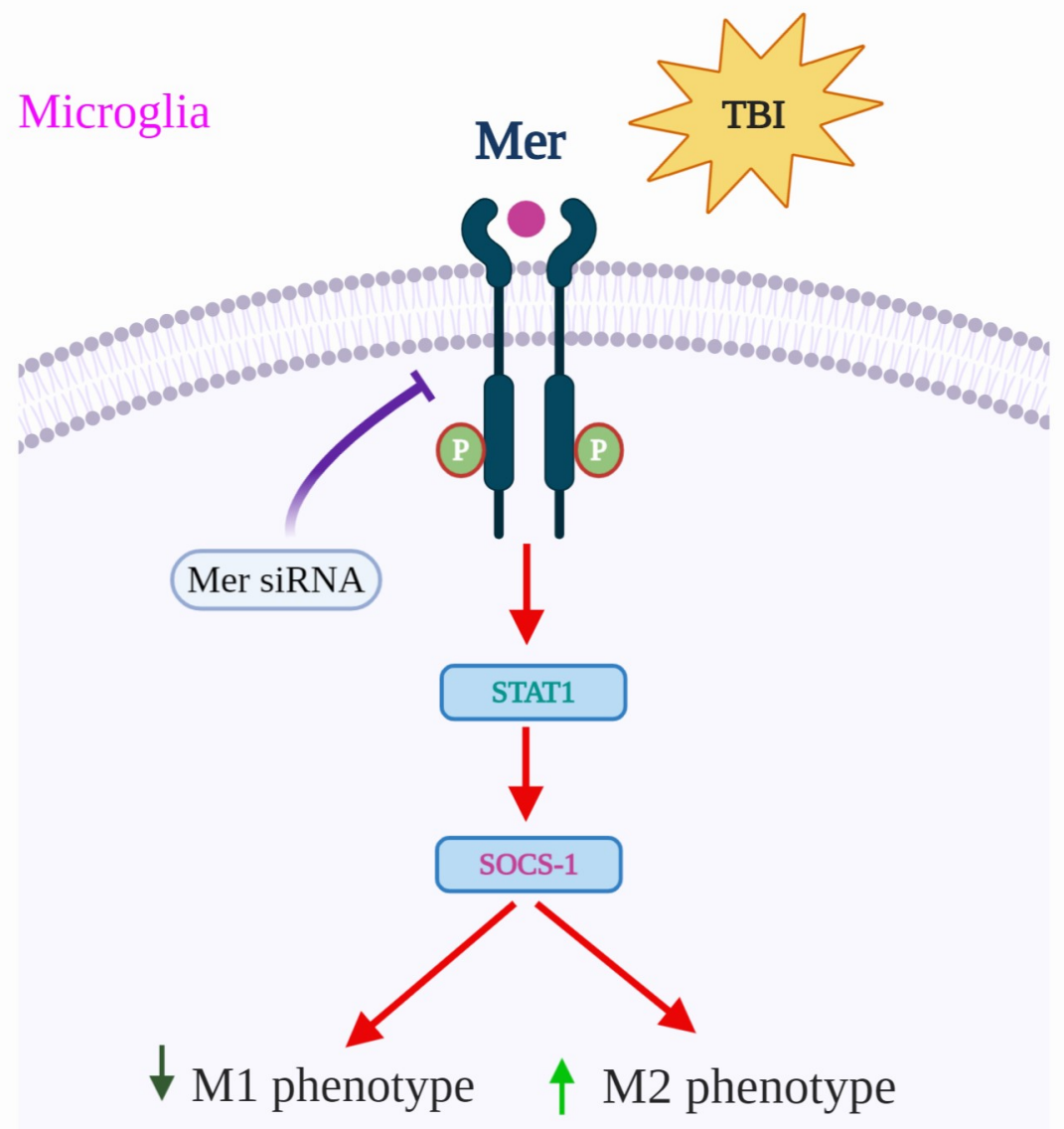

Figure 8. The proposed mechanism of Mer in regulating microglial M1/M2 polarization and neuroinflammation following TBI via activation of the STAT1/SOCSs signaling pathway. 


\section{Supplementary data}

A
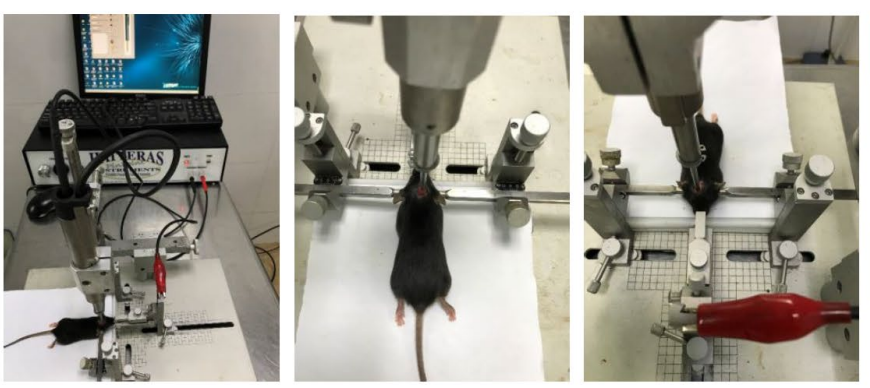

\begin{tabular}{|l|l|l|}
\hline & Experimental parameters \\
\hline Impact tip diameter & $3 \mathrm{~mm}$ \\
\hline Impact velocity & $3 \mathrm{~m} / \mathrm{s}$ \\
\hline Impact duration & $0.15 \mathrm{~s}$ \\
\hline Impact depth & $2 \mathrm{~mm}$ \\
\hline
\end{tabular}

B

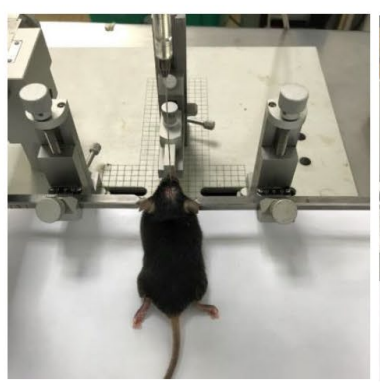

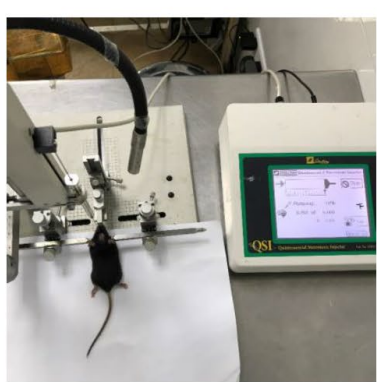

\begin{tabular}{|l|l|}
\hline & \multicolumn{1}{c}{\begin{tabular}{c} 
Experimental parameters \\
\hline Stereotactic location
\end{tabular}} \\
\hline Injection volume & $2.0 \mathrm{~mm}$ lateral of the bregma, $2.5 \mathrm{~mm}$ deep below the skull \\
\hline Injection velocity & $0.5 \mu \mathrm{l} / \mathrm{min}$ \\
\hline
\end{tabular}

Supplementary Figure 1. (A) The controlled cortical impact (CCI) model and experimental parameters of traumatic brain injury (TBI) in mice. (B) The intracerebroventricular injection and experimental parameters in mice. 
A Expression profiles of Mer and M1/M2 polarization markers following TBI

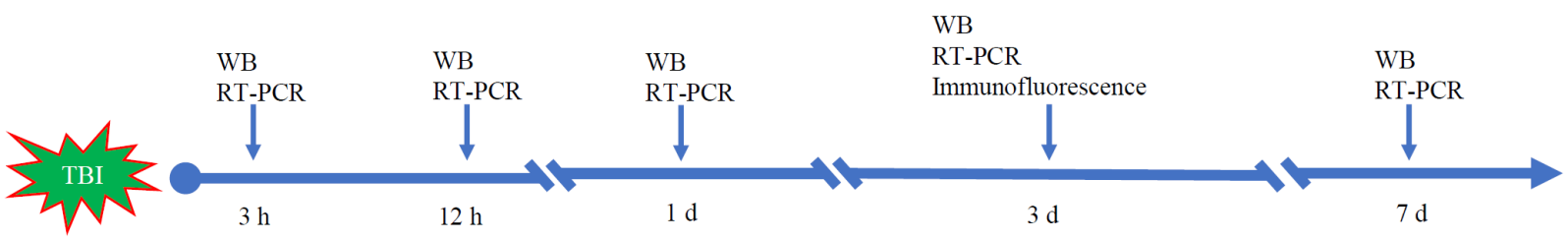

Groups: Sham, TBI 3 h, TBI 12 h, TBI 1 d, TBI 3 d, TBI $7 \mathrm{~d}$.

B The effect of Mer knockdown on microglial M1/M2 polarization and neuroinflammation following TBI

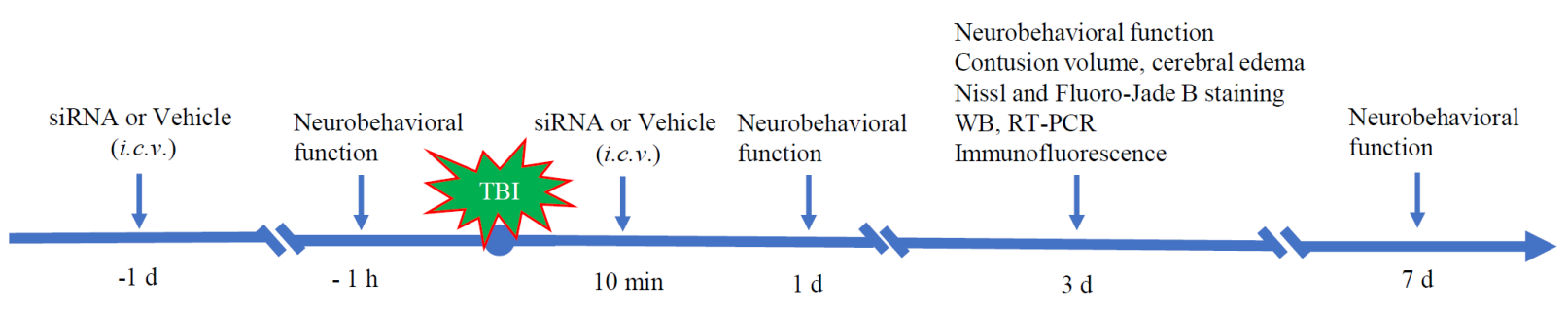

Groups: Sham, TBI + Vehicle, TBI + Control siRNA, TBI + Mer siRNA.

C The effect of PS on regulating STAT1/SOCSs pathway following TBI

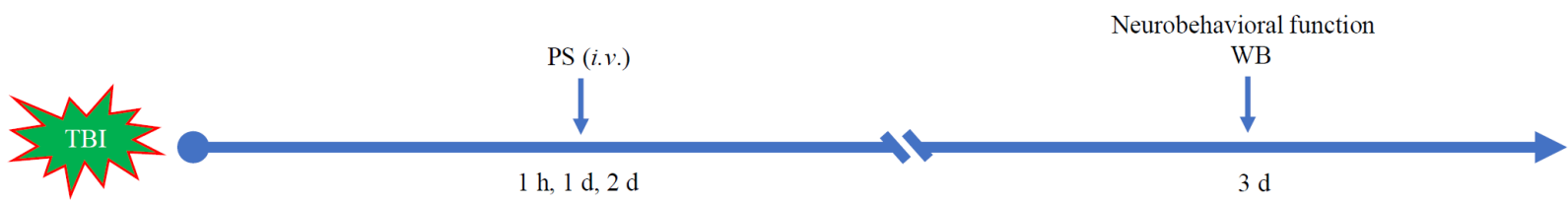

Groups: Sham, TBI + Vehicle, TBI + recombinant protein S (PS).

D Mer knockdown abolished the effect of PS following TBI

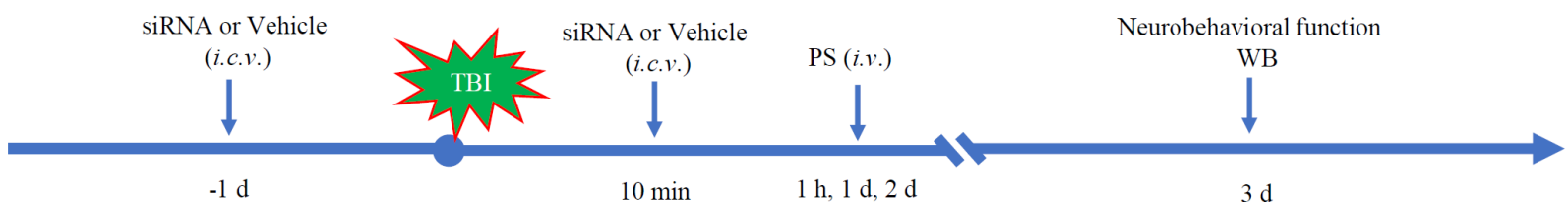

Groups: TBI + Vehicle, TBI + PS + Control siRNA, TBI + PS + Mer siRNA

Supplementary Figure 2. Experimental design and animal groups. (A) Western blot (WB) and Real-Time Polymerase Chain Reaction (RT-PCR) was performed to evaluate the expression profiles of Mer and M1/M2 polarization markers at different time points after TBI (including $3 \mathrm{~h}, 12 \mathrm{~h}, 1 \mathrm{~d}, 3 \mathrm{~d}$, and $7 \mathrm{~d}$ ), as well as in the sham group; Besides, immunofluorescence was performed on day 3 after injury. (B) An in vivo knockdown of Mer siRNA was adopted to evaluate the role of Mer in regulating microglial M1/M2 polarization after TBI. Mice were randomly distributed into sham, TBI + Vehicle, TBI + Control siRNA, and TBI + Mer siRNA groups. Intracerebroventricular injection (i.c.v.) of siRNA was performed $1 \mathrm{~d}$ before and $10 \mathrm{~min}$ after TBI. The neurobehavioral functions were assessed before as well as 1, 3, $7 \mathrm{~d}$ after TBI. The peri-injured cerebral cortex from each group and the equivalent area in the sham-operated mice was collected for RT-PCR, WB, and immunohistochemistry analysis at $3 \mathrm{~d}$ after TBI. (C) To evaluate the effect of PS on regulating STAT1/SOCSs pathway after TBI, mice were randomly distributed into Sham, TBI + Vehicle, and TBI + recombinant protein $\mathrm{S}$ (PS) groups. PS $(0.2 \mathrm{mg} / \mathrm{kg})$ was administered via the tail vein at $1 \mathrm{~h}, 1 \mathrm{~d}$, and $2 \mathrm{~d}$ after the CCI. WB and 
neurobehavioral assessments were conducted on day 3 post-injury. (D) Mice were randomly distributed into TBI + Vehicle, TBI + PS + Control siRNA, and TBI + PS + Mer siRNA groups. Mer siRNA (i.c.v.) was administrated $1 \mathrm{~d}$ before and 10 min after TBI, and PS was administered via the tail vein at $1 \mathrm{~h}, 1 \mathrm{~d}$, and $2 \mathrm{~d}$ after the injury. WB and neurobehavioral assessments were conducted on day 3 post-injury. 


\section{Figures}

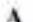

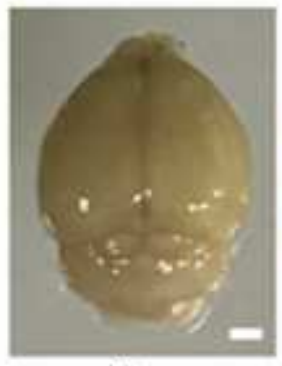

Sham

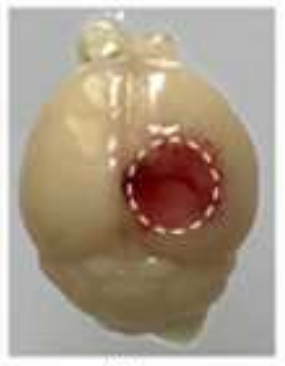

3h

B

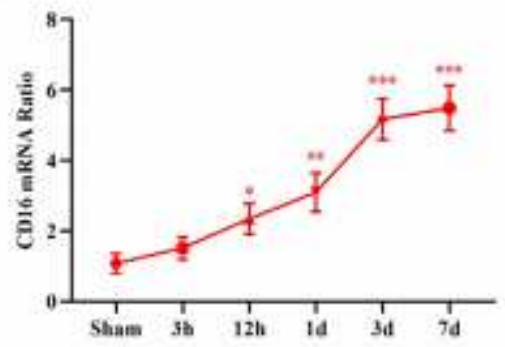

E

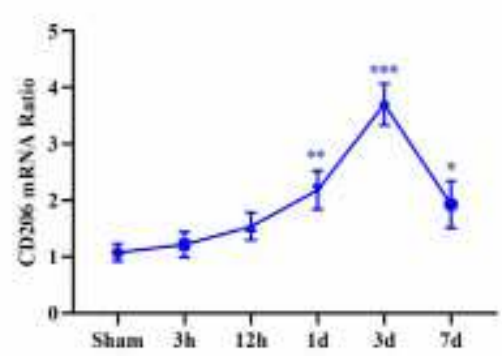

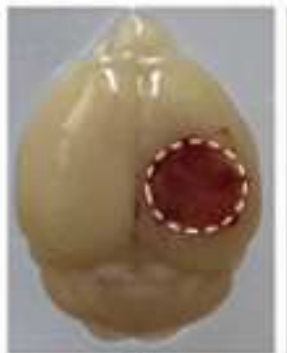

12h

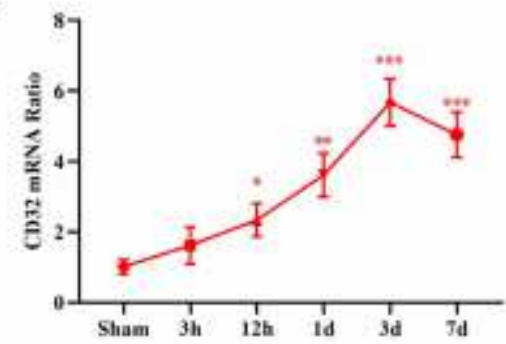

F

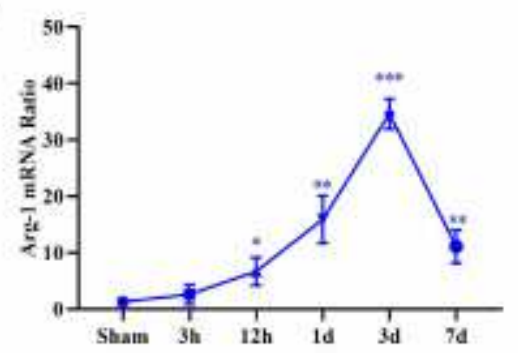

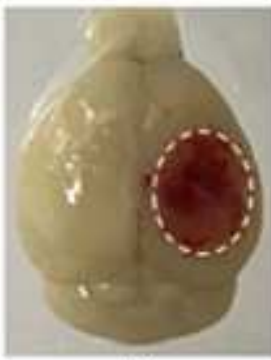

3d

D

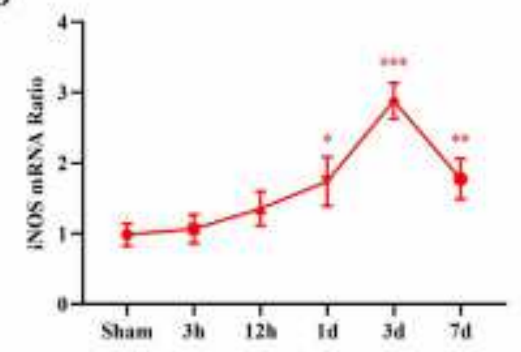

G

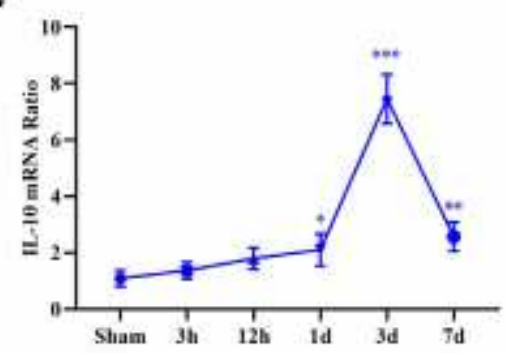

\section{Figure 1}

Dynamic changes in mRNA expression of microglial M1 and M2 phenotypic markers following TBI. (A) Representative photographs of whole brains in the sham and different traumatic brain injury (TBI) groups (at $3 \mathrm{~h}, 12 \mathrm{~h}, 1 \mathrm{~d}, 3 \mathrm{~d}$, and $7 \mathrm{~d}$ post-insult, respectively). Scale bar $=1 \mathrm{~mm}$. Quantitative ReverseTranscription Polymerase Chain Reaction (RT-PCR) was used to assess the mRNA expression levels of microglial M1 and M2 phenotypic markers in the injured cortex at $3 \mathrm{~h}, 12 \mathrm{~h}, 1 \mathrm{~d}, 3 \mathrm{~d}$, and $7 \mathrm{~d}$ after $\mathrm{TBI}$ or the equivalent area of the sham-operated brains. (B-D) Expressions of mRNA of M1 phenotypic markers, including CD16 (B), CD32 (C), and iNOS (D), were gradually increased over time from $12 \mathrm{~h}$ onward and remained elevated for at least $7 \mathrm{~d}$ after injury. (E-G) Expressions of mRNA of M2 phenotypic markers, including CD206 (E), Arg-1 (F), and IL-10 (G), were significantly up-regulated at $1 \mathrm{~d}, 12 \mathrm{~h}$, and $1 \mathrm{~d}$ after TBI, respectively, and all decreased at $7 \mathrm{~d}$ post-injury. Data are expressed as fold change compared to sham-operated controls. $\mathrm{n}=6$ mice per group. ${ }^{*}, \mathrm{p}<0.05 ;{ }^{* \star}, \mathrm{p}<0.01 ;{ }^{* \star \star}, \mathrm{p}<0.001$. one-way ANOVA followed by Bonferroni's post-hoc tests. 
A
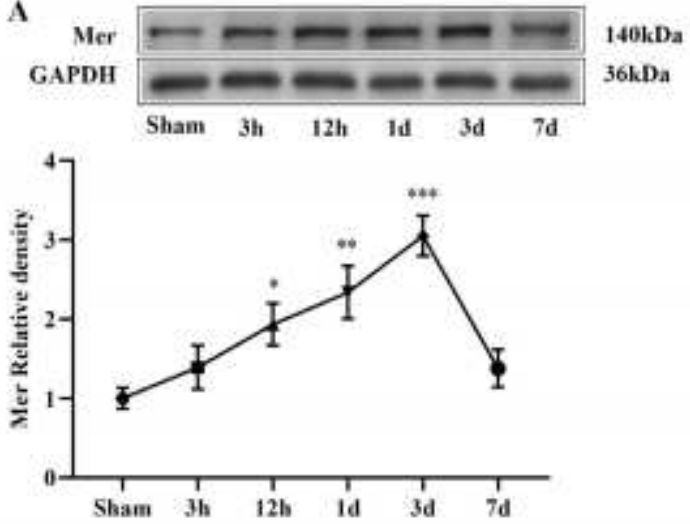

B

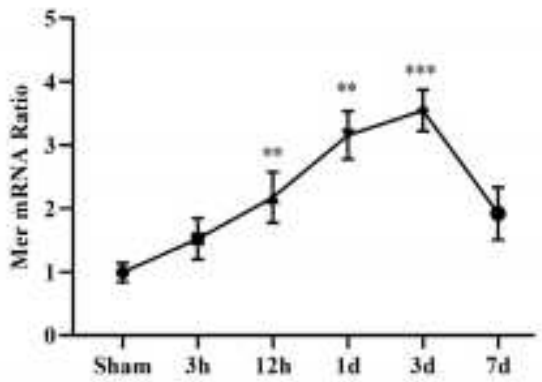

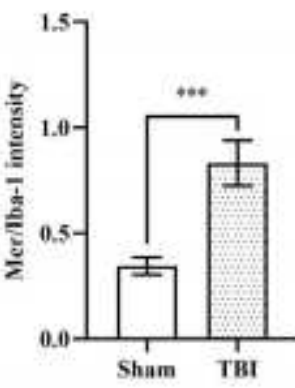

D

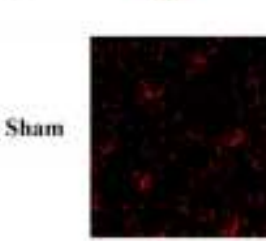

Iba-1

DAPI
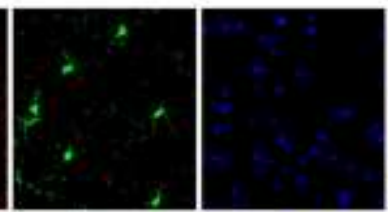

Merge

TBI
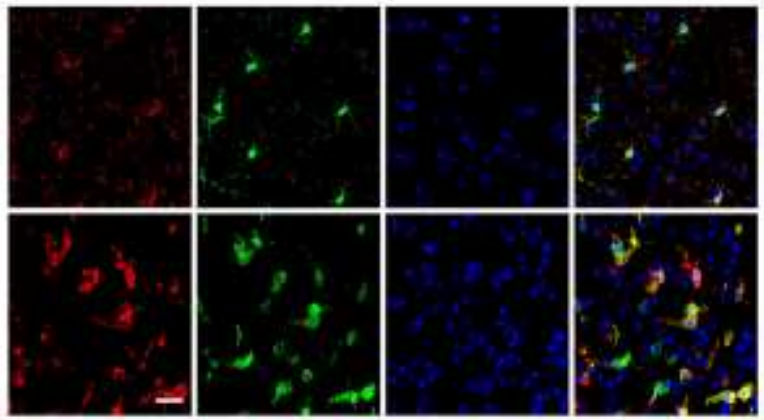

E

Mer

Tha-1

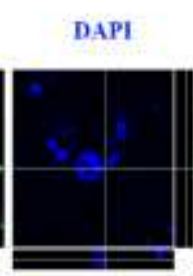

Merge
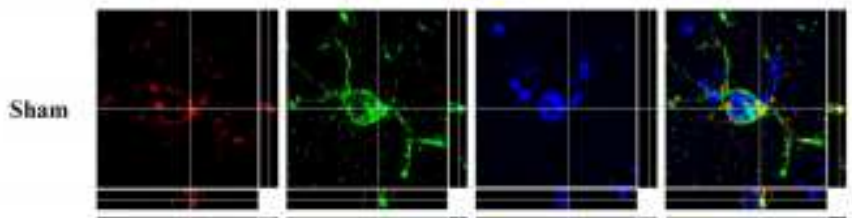

TBI
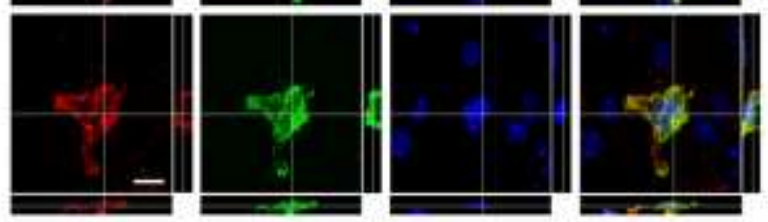

\section{Figure 2}

Expression patterns and cellular localization of Mer following TBI. (A) Representative immunoblots and quantification showing the expression level of Mer protein in the injured cortex at $3 \mathrm{~h}, 12 \mathrm{~h}, 1 \mathrm{~d}, 3 \mathrm{~d}$, and 7 $\mathrm{d}$ after TBI or the equivalent area of the sham-operated brains. Data are expressed as fold change compared to sham-operated controls. $n=6$ mice per group. (B) Quantitative RT-PCR was used to assess the mRNA expression level of Mer in the injured cortex at $3 \mathrm{~h}, 12 \mathrm{~h}, 1 \mathrm{~d}, 3 \mathrm{~d}$, and $7 \mathrm{~d}$ after TBI or the equivalent area of the sham-operated brains. Data are expressed as fold change compared to shamoperated controls. $\mathrm{n}=6$ mice per group. Double immunofluorescent staining of Mer with the microglial marker Iba-1 in the ipsilateral cerebral cortex was performed at $3 \mathrm{~d}$ post-TBI or sham-operation. (C) Representative confocal images from the ipsilateral cortex showing Mer was abundantly expressed in the plasma membrane of microglia and substantially upregulated in activated microglia following TBI. Scale bar $=15 \mu \mathrm{m}$. (D) Fluorescence intensity quantification of Mer expression in activated microglia in the impacted cortical area 3 days after TBI, compared to that in resting microglia from sham-operated brains. $\mathrm{n}=6$ mice per group. (E) Confocal microscopy analysis at a single-cell resolution showing Mer expression was substantially upregulated in the activated microglia with phagocytotic morphology at $3 \mathrm{~d}$ post-TBI. Orthogonal views demonstrated the colocalization of Mer and Iba- 1 in microglia. Scale bar $=5$ $\mu \mathrm{m}$. In A-B, data are presented as mean $\pm \mathrm{SD} ;{ }^{*}, \mathrm{p}<0.05 ; * *, \mathrm{p}<0.01 ; * \star *, p<0.001$. one-way ANOVA followed by Bonferroni's post-hoc tests. In $D$, data are presented as Mean $\pm S D ; \star \star \star, p<0.001$ by Student's t-test. 
A
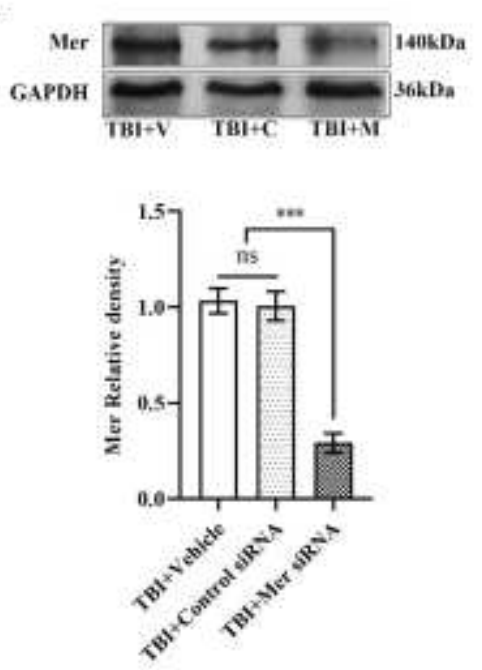

B

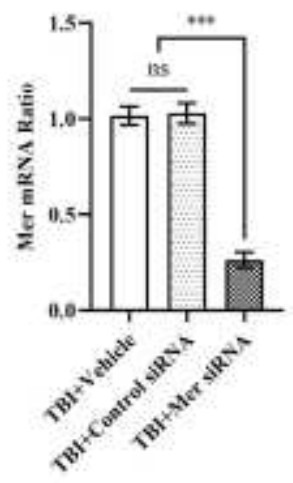

C

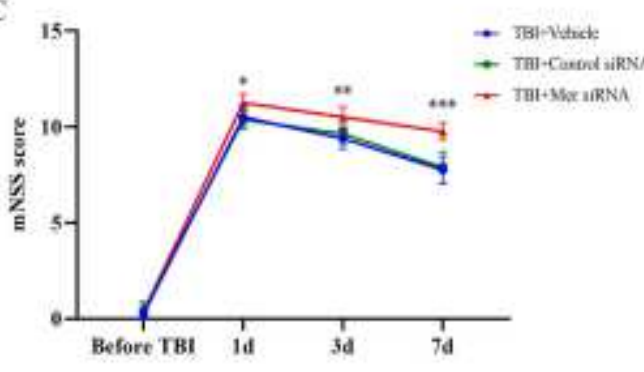

D

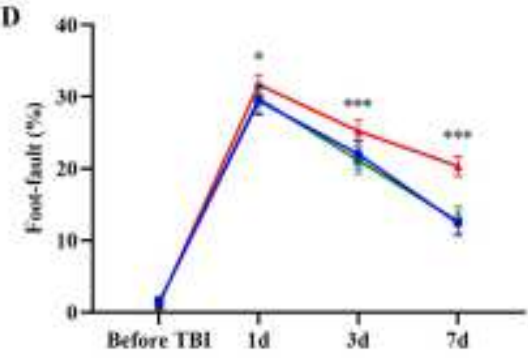

E

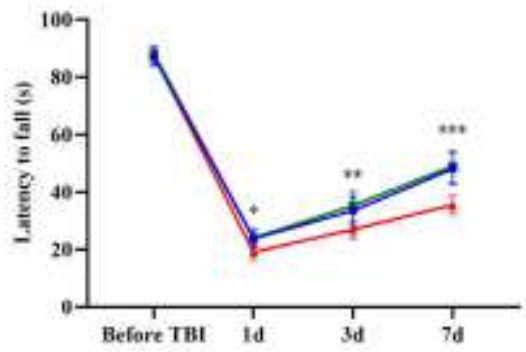

$\mathbf{F}$
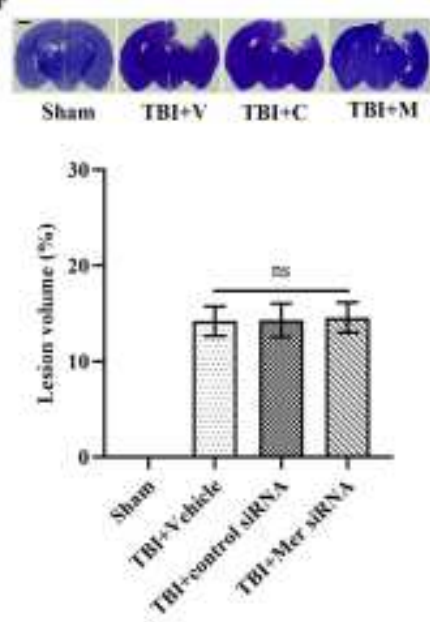

G

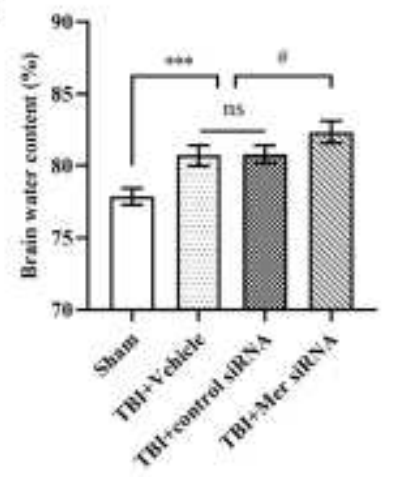

\section{Figure 3}

In vivo knockdown of Mer worsened the functional outcomes after TBI. (A) Western blot was used to assess the knockdown efficacy of Mer siRNA. Representative immunoblots and quantification showing Mer siRNA inhibited the expression of Mer protein in the injured cortex at $3 \mathrm{~d}$ post-TBI. TBI + Vehicle: TBI + $\mathrm{V}, \mathrm{TBI}+$ Control siRNA: TBI + C, TBI + Mer siRNA: TBI + M. GAPDH: loading control. Data are expressed as fold change compared to the TBI + V group; $n=6$ mice per group. (B) Quantitative RT-PCR analysis also showing Mer siRNA significantly inhibited the expression of Mer mRNA in the injured cortex at $3 \mathrm{~d}$ postTBI. Data are expressed as fold change compared to the TBI + V group; $n=6$ mice per group. (C-E) Modified neurological severity scores (mNSS) (C), foot-fault test (D), and rotarod test (E) performed before and 1, 3, and $7 \mathrm{~d}$ after TBI. $\mathrm{n}=8$ mice per group. (F) Quantification of TBI-induced lesion volume at $3 \mathrm{~d}$ post-insult. $\mathrm{n}=6$ mice per group. Scale bar $=1 \mathrm{~mm}$. (G) Cerebral edema was measured by brain water content. Mer siRNA significantly elevated brain edema level at $3 \mathrm{~d}$ post-injury, when compared to both the Vehicle and Control siRNA group. $n=8$ mice per group. In A-G, data are presented as mean $\pm S D ; *, \#, p<$ $0.05 ; * \star, p<0.01, * \star *, p<0.001$, ns, nonsignificant, $p>0.05$. one-way ANOVA followed by Bonferroni's post-hoc tests. 


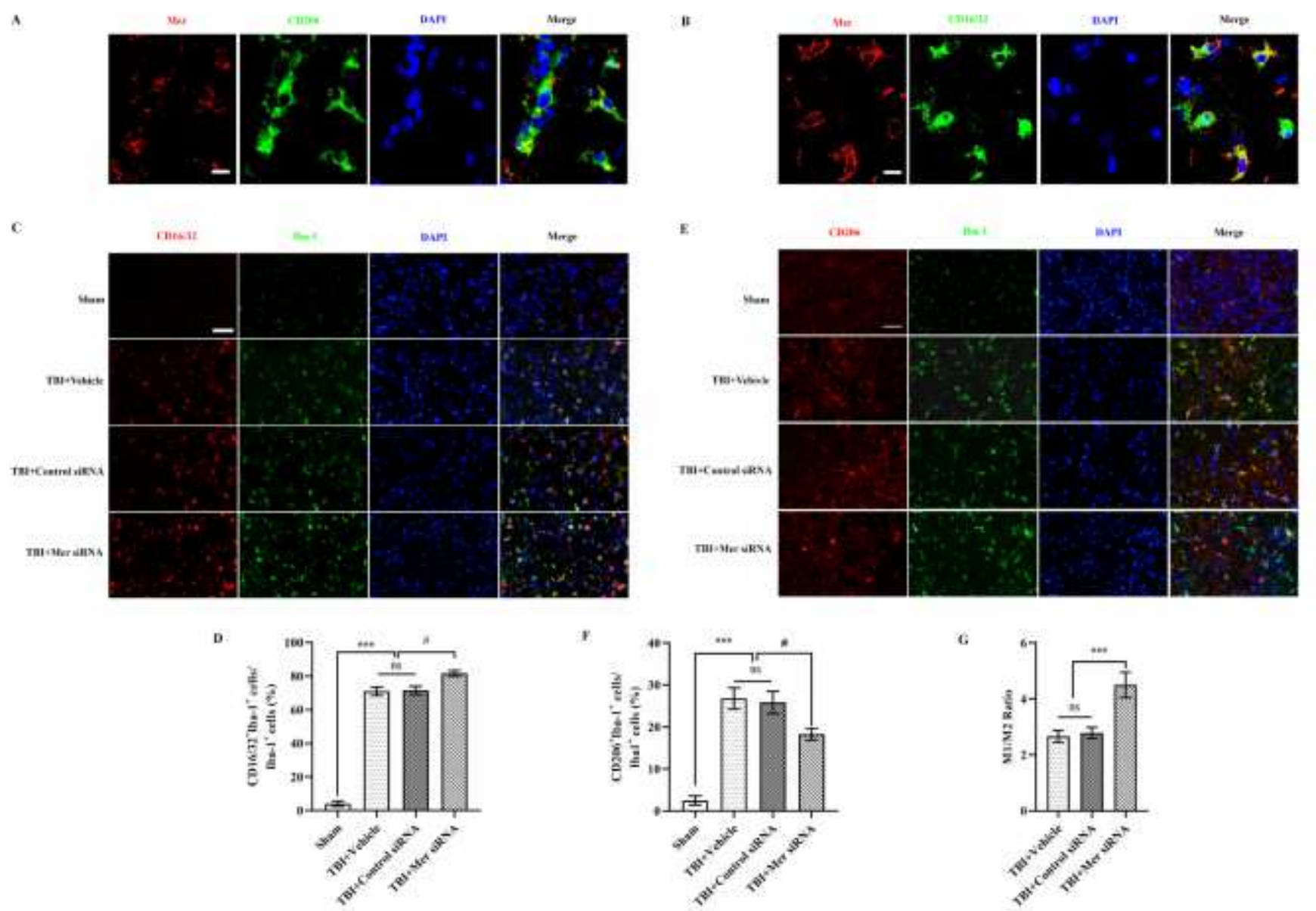

\section{Figure 4}

Mer modulated microglial M1/M2 polarization after TBI. (A) Representative images of immunofluorescent staining for Mer (red), CD16/32 (green) and DAPI (blue) showing Mer was expressed in CD16/32-positive cells in the perilesional area of cortex at $3 \mathrm{~d}$ post-TBI. Scale bar $=20 \mu \mathrm{m}$. (B) Representative images of immunofluorescent staining for Mer (red), CD206 (green) and DAPI (blue) showing Mer was expressed in CD206-positive cells in the perilesional area of cortex at $3 \mathrm{~d}$ post-TBI. Scale bar $=20 \mu \mathrm{m}$. (C) Representative images of immunofluorescent staining for CD16/32 (red), Iba-1 (green) and DAPI (blue) in the ipsilateral cortex at $3 \mathrm{~d}$ post-TBI. Scale bar $=50 \mu \mathrm{m}$. (D) Quantification showing the percentage of CD16/32 and Iba-1 double-positive cells was significantly increased in the cortex at day 3 after TBI, which was further elevated in the Mer siRNA group. $n=6$ mice per group. (E) Representative images of immunofluorescent staining for Mer (red), CD206 (green) and DAPI (blue) in the ipsilateral cortex at $3 \mathrm{~d}$ post-TBI. Scale bar $=50 \mu \mathrm{m}$. (F) Quantification showing the percentage of CD206 and Iba-1 double-positive cells increased significantly in the ipsilateral cortex at day 3 after TBI, however, it significantly decreased following Mer siRNA administration. (G) The ratio between CD16/32+ Iba-1+ M1 cells and CD206+ Iba-1+ M2 cells nearly doubled after Mer siRNA administration. In D, F, and G, data are presented as mean $\pm S D ; \#, p<0.05 ; * \star \star, p<0.001 ; n s$, non-significant, $p>0.05$. one-way ANOVA followed by Bonferroni's post-hoc tests. 

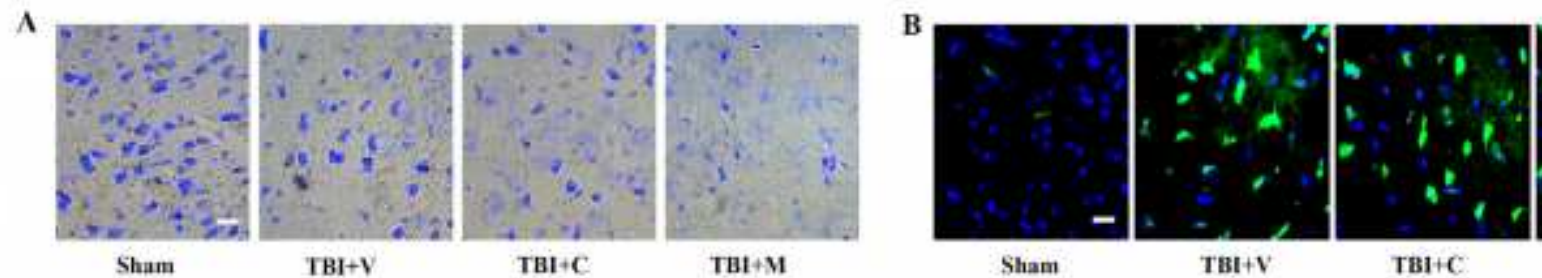

Sham

$T B I+V$

$\mathrm{TB1}+\mathrm{C}$.

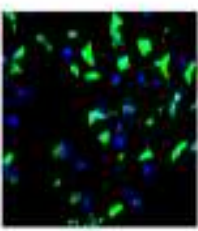

$\mathrm{TBI}+\mathrm{M}$
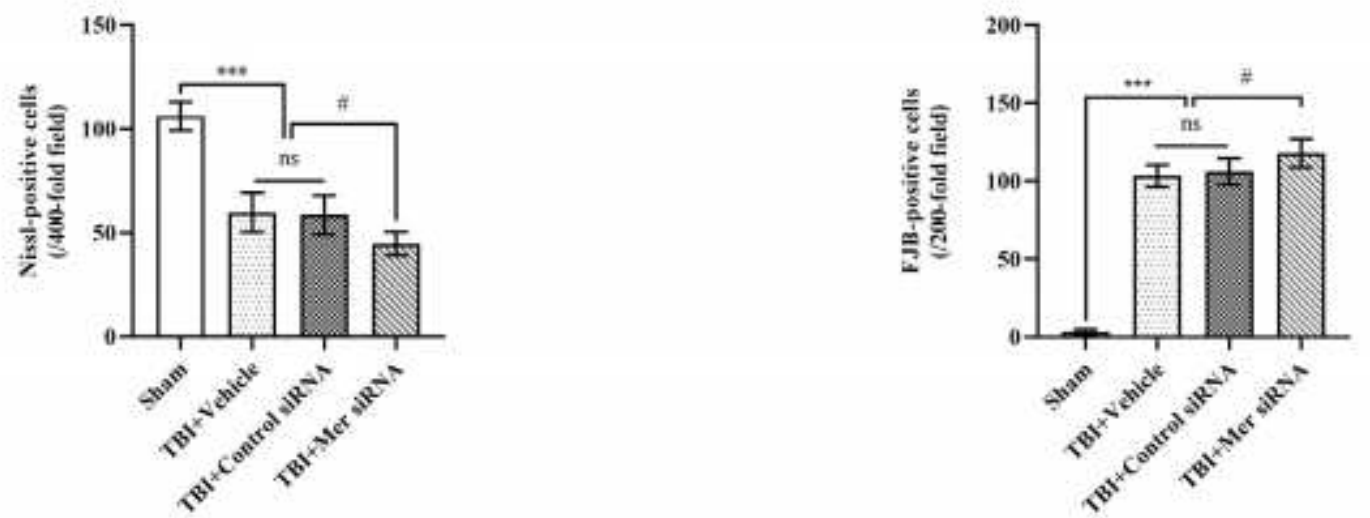

\section{Figure 5}

Inhibition of Mer aggravated neuronal damage and degeneration following TBI. (A) Representative images of Nissl staining in the ipsilateral cortex from the sham, TBI + Vehicle (TBI + V), TBI + Control siRNA $(T B I+C)$ and TBI + Mer siRNA $(T B I+M)$ groups, respectively. Quantification analysis showing TBI caused a significant decrease in the number of Nissl-positive cells in the ipsilateral cortex at $3 \mathrm{~d}$ post-TBI, and Mer siRNA application further decreased the number of Nissl-positive cells in the injured cortex after TBI. $n=6$ mice per group. Scale bar $=20 \mu \mathrm{m}$. (B) Representative images of Fluoro-Jade B (FJB) staining in the ipsilateral cortex from the sham, $\mathrm{TBI}+\mathrm{V}, \mathrm{TBI}+\mathrm{C}$, and $\mathrm{TBI}+\mathrm{M}$ groups, respectively. Quantification analysis showing TBI caused a significant increase in the number of FJB-positive cells in the ipsilateral cortex at $3 \mathrm{~d}$ postTBI, and Mer siRNA application further increased the number of FJB-positive cells in the injured cortex after TBI. $n=6$ mice per group. Scale bar $=20 \mu \mathrm{m}$. In A-B, data are presented as Mean \pm SD; $\#, p<0.05 ; * \star *, p<0.001 ; n s$, non-significant, $p>0.05$. one-way ANOVA followed by Bonferroni's post-hoc tests. 
A
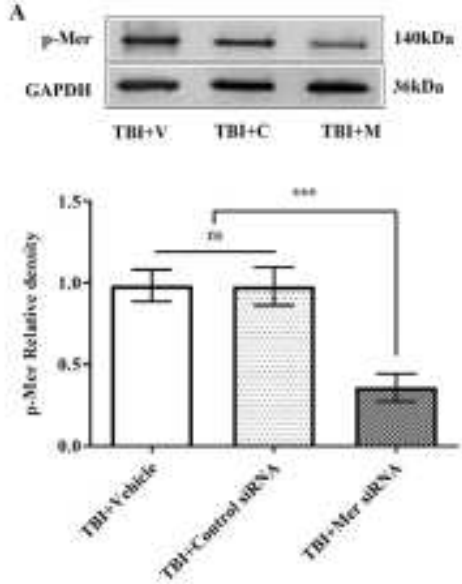

D

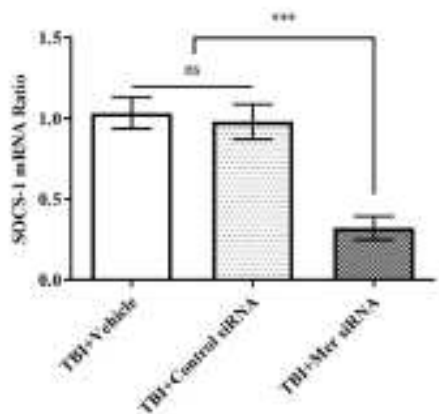

B
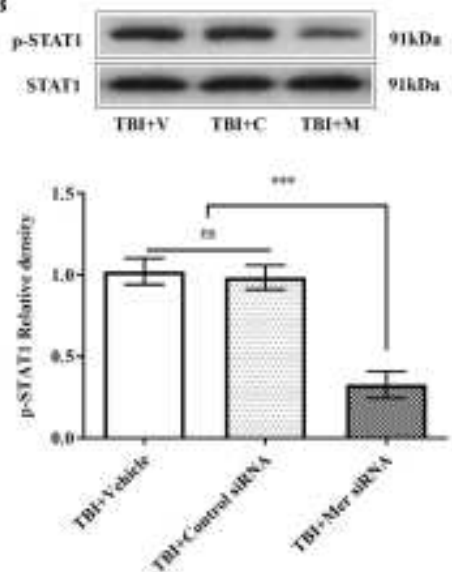

E

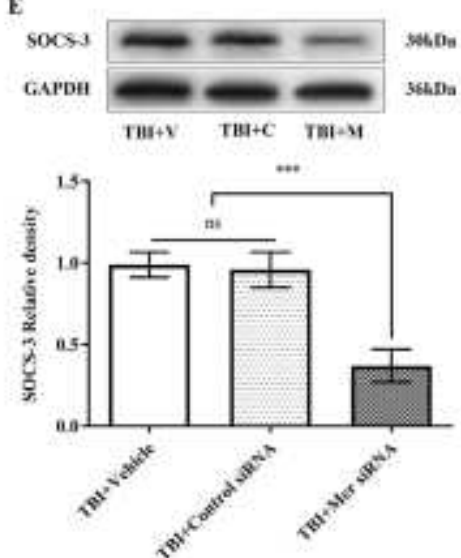

C
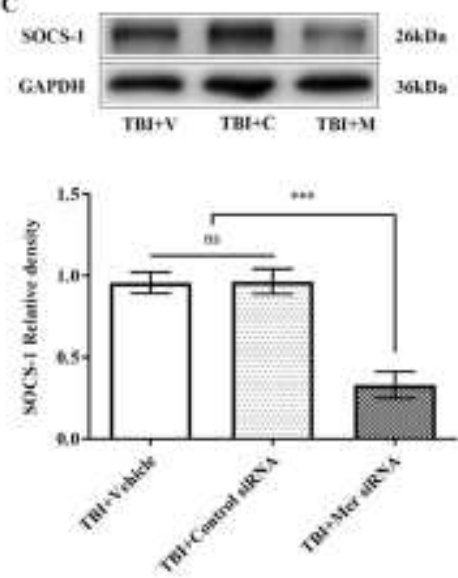

F

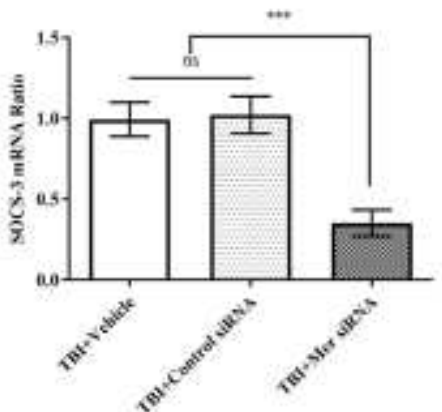

\section{Figure 6}

Inhibition of Mer reduced STAT1 activation and SOCSs expression following TBI. (A, B, C, E) Representative immunoblots and quantification showing the expression of phosphorylated Mer ( $\mathrm{p}-\mathrm{Mer}$ ) (A), phosphorylated STAT1 (p-STAT1) (B), SOCS-1 (C) and SOCS-3 (E) was significantly inhibited by Mer siRNA administration on day 3 following TBI. TBI + Vehicle: TBI + V, TBI + Control siRNA: TBI + C; TBI + Mer siRNA: TBI + M. GAPDH: loading control. Data are expressed as fold change compared to the TBI + V group; $\mathrm{n}=6$ mice per group. (D, F) Quantitative RT-PCR analysis showing Mer siRNA application significantly inhibited the mRNA expression of SOCS-1 (D) and SOCS-3 (F) in the injured cortex at $3 \mathrm{~d}$ post-TBI. Data are expressed as fold change compared to the TBI + V group; $n=6$ mice per group. In A-F, data are presented as Mean $\pm S D$; ns, non-significant, $p>0.05$; $\star \star \star ~ p<0.001$. one-way ANOVA followed by Bonferroni's post-hoc tests. 

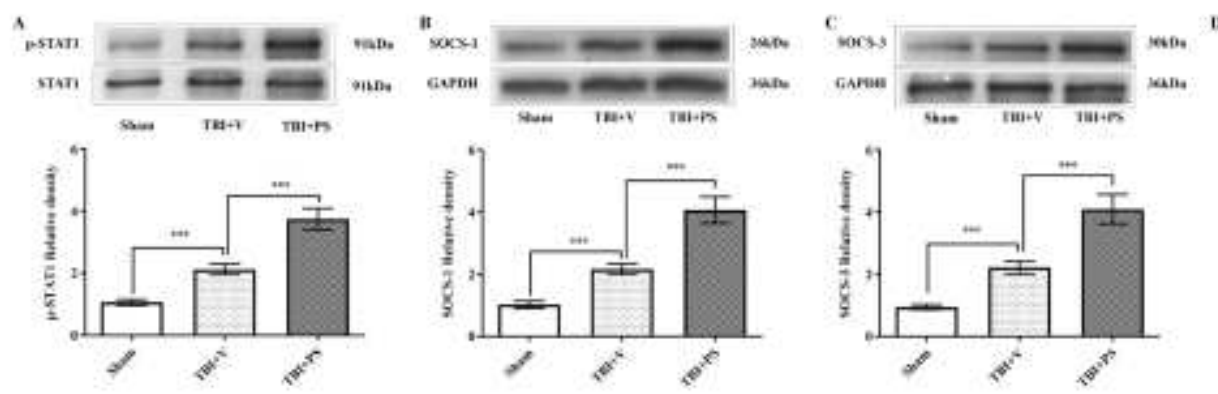

D
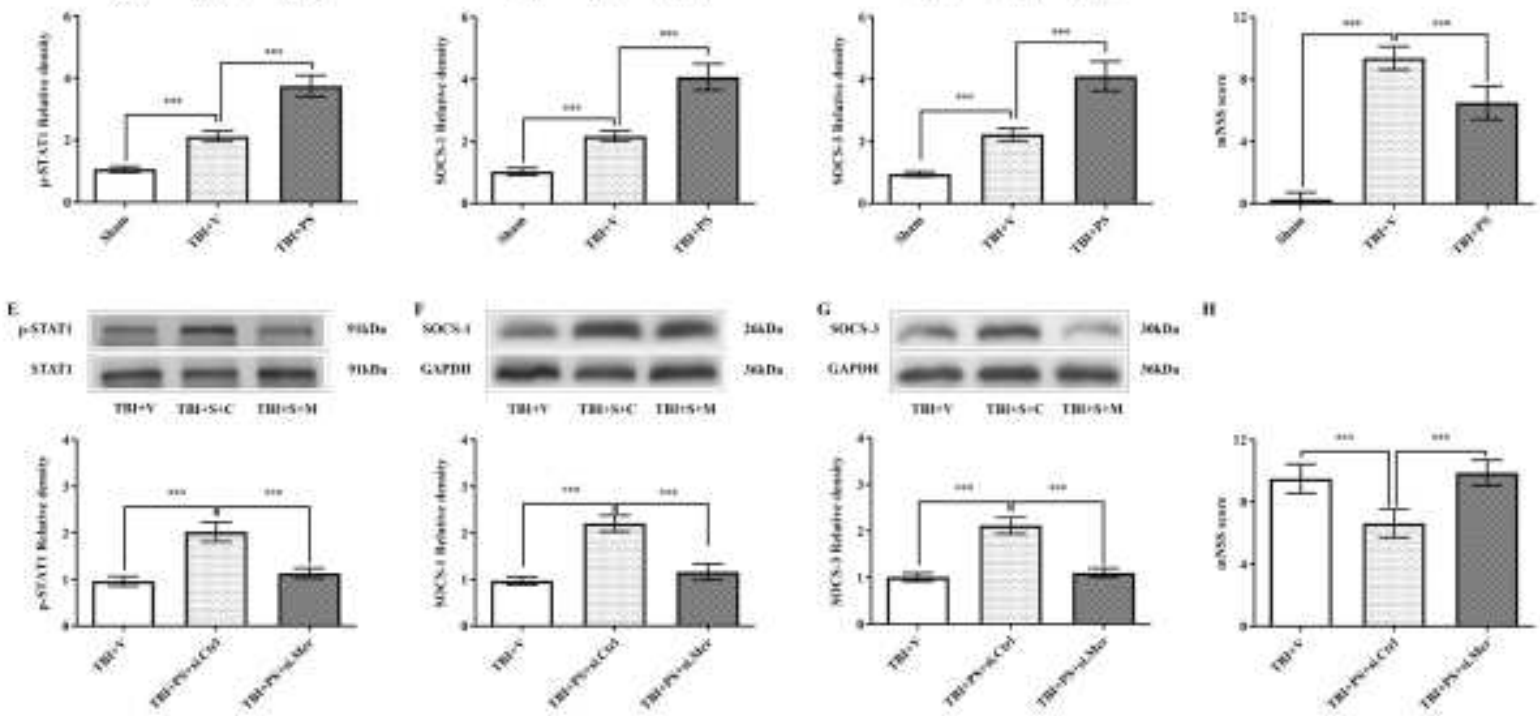

Figure 7

Mer activation alleviated functional deficits following TBI. (A-C) Representative immunoblots and quantification showing protein expression of p-STAT1 (A), SOCS-1 (B), and SOCS-3 (C) in the injured cortex at 3 d post-TBI; TBI + Vehicle: TBI + V, TBI + recombinant protein S: TBI + PS. GAPDH: loading control. Data are expressed as fold change compared to the sham group; $n=6$ mice per group. (D) Modified neurological severity scores (mNSS) performed at $3 \mathrm{~d}$ after TBI. $\mathrm{n}=8$ mice per group. (E-G) Representative immunoblots and quantification showing protein expression of p-STAT1 (E), SOCS-1 (F), and SOCS-3 (G) in the injured cortex at $3 \mathrm{~d}$ after TBl; TBI + Vehicle: TBI + V, TBI + PS + Control siRNA (si.Ctrl): TBI + S + C, TBI + S + Mer siRNA (si.Mer): TBI + S +M. GAPDH: loading control. Data are expressed as fold change compared to the TBI $+V$ group; $n=6$ mice per group. $(H)$ The mNSS performed at $3 \mathrm{~d}$ after TBI. $\mathrm{n}=8$ mice per group. In $A-H$, data are presented as Mean $\pm S D ; * \star \star, p<0.001$. one-way ANOVA followed by Bonferroni's post-hoc tests. 


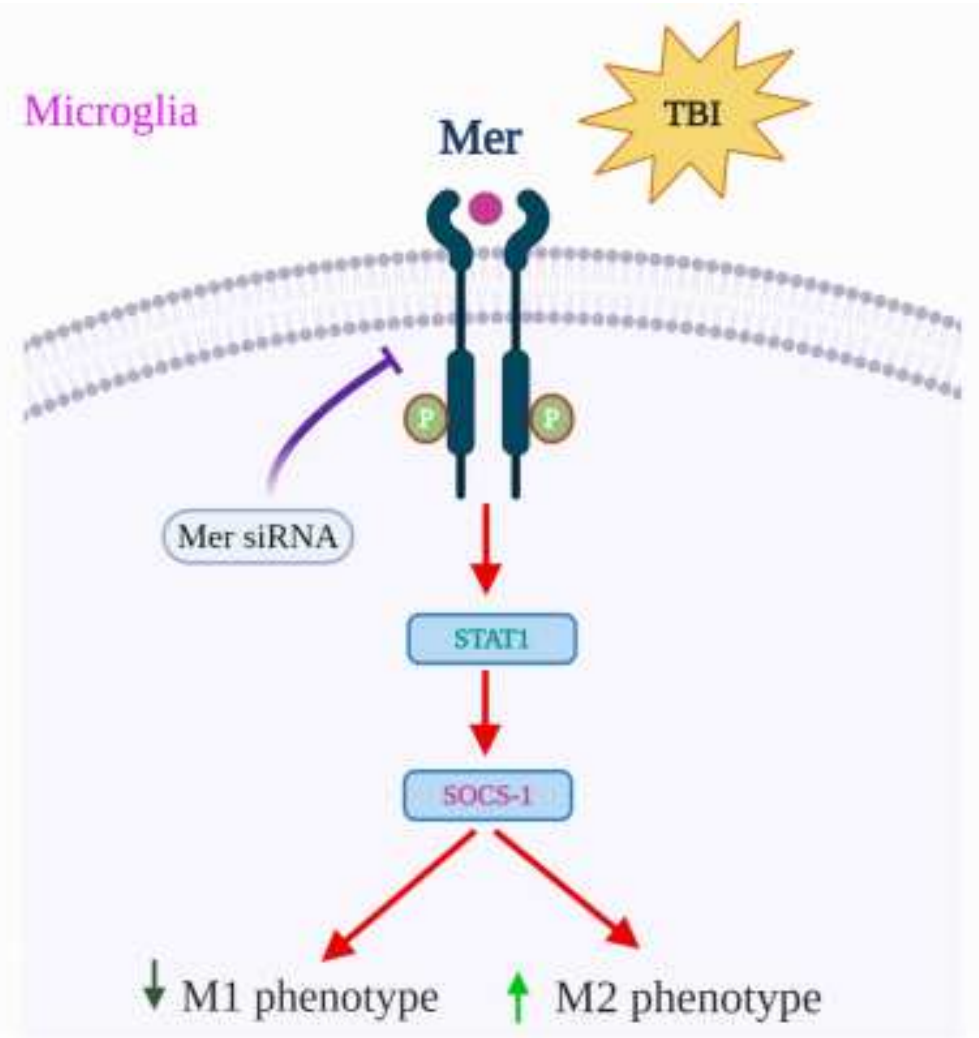

Figure 8

The proposed mechanism of Mer in regulating microglial M1/M2 polarization and neuroinflammation following TBI via activation of the STAT1/SOCSs signaling pathway. 